\title{
THE LEE-YANG AND PÓLYA-SCHUR PROGRAMS. I. LINEAR OPERATORS PRESERVING STABILITY
}

\author{
JULIUS BORCEA AND PETTER BRÄNDÉN
}

\begin{abstract}
In 1952 Lee and Yang proposed the program of analyzing phase transitions in terms of zeros of partition functions. Linear operators preserving non-vanishing properties are essential in this program and various contexts in complex analysis, probability theory, combinatorics, and matrix theory. We characterize all linear operators on finite or infinite-dimensional spaces of multivariate polynomials preserving the property of being non-vanishing whenever the variables are in prescribed open circular domains. In particular, this solves the higher dimensional counterpart of a long-standing classification problem originating from classical works of Hermite, Laguerre, Hurwitz and PólyaSchur on univariate polynomials with such properties.
\end{abstract}

\section{Contents}

Introduction

1. Operator Symbols and $\Omega$-Stable Polynomials

1.1. Algebraic and Transcendental Symbols

1.2. Fundamental Properties of Stable Polynomials

2. Hard-Core/Algebraic Classification: Sufficiency

2.1. Multi-affine Polynomials and the Lieb-Sokal Lemma

2.2. Decoupling Schemes: Polarizations of Polynomials and Operators 10

3. Algebraic Classification: Necessity in the Complex Case 12

4. Real Stability Preservers 13

5. Soft-Core/Transcendental Classifications 15

5.1. Generalized Jensen Multipliers

5.2. Multivariate Szász Principles 16

5.3. Proof of Theorem $5.1 \quad 19$

6. Circular Domains and Lee-Yang Polynomials 20

6.1. Products of Open Circular Domains 20

6.2. Linear Preservers of Lee-Yang Polynomials 23

7. Related Results 24

8. Further Directions 25

Acknowledgments 25

References

2000 Mathematics Subject Classification. Primary: 47B38; Secondary: 05A15, 05C70, 30C15, 32A60, 46E22, 82B20, 82B26.

Key words and phrases. Phase transitions, Lee-Yang theory, Pólya-Schur theory, linear operators, polarization, stable polynomials, graph polynomials, symmetrization, apolarity, Szász principles, multiplier sequences.

The first author is supported by the Royal Swedish Academy of Sciences. The second author is supported by the Göran Gustafsson Foundation. 


\section{INTRODUCTION}

Zero loci of multivariate polynomials or transcendental entire functions and their dynamics under linear transformations are central topics in geometric function theory which in recent years has found applications in statistical mechanics, combinatorics, probability theory and matrix theory [6, 8, 10, 11, 12, 13, 153. For instance, as shown in 6, 13, 33, the Lee-Yang program on phase transitions in equilibrium statistical mechanics [31, 59] is intimately related to the problems of describing linear operators on polynomial spaces that preserve the property of being nonvanishing when the variables are in prescribed subsets of $\mathbb{C}^{n}$. In this paper we fully solve these problems for (products of) open circular domains. In particular, this accomplishes a long-standing classification program originating from classical works of Hermite, Laguerre, Hurwitz and Pólya-Schur on univariate polynomials with such properties [14, 17, 28, 41, 42, 43.

Given an integer $n \geq 1$ and $\Omega \subset \mathbb{C}^{n}$ we say that $f \in \mathbb{C}\left[z_{1}, \ldots, z_{n}\right]$ is $\Omega$-stable if $f\left(z_{1}, \ldots, z_{n}\right) \neq 0$ whenever $\left(z_{1}, \ldots, z_{n}\right) \in \Omega$. A $\mathbb{K}$-linear operator $T: V \rightarrow$ $\mathbb{K}\left[z_{1}, \ldots, z_{n}\right]$, where $\mathbb{K}=\mathbb{R}$ or $\mathbb{C}$ and $V$ is a subspace of $\mathbb{K}\left[z_{1}, \ldots, z_{n}\right]$, is said to preserve $\Omega$-stability if for any $\Omega$-stable polynomial $f \in V$ the polynomial $T(f)$ is either $\Omega$-stable or $T(f) \equiv 0$. For $\kappa=\left(\kappa_{1}, \ldots, \kappa_{n}\right) \in \mathbb{N}^{n}$ let $\mathbb{K}_{\kappa}\left[z_{1}, \ldots, z_{n}\right]=\{f \in$ $\left.\mathbb{K}\left[z_{1}, \ldots, z_{n}\right]: \operatorname{deg}_{z_{i}}(f) \leq \kappa_{i}, 1 \leq i \leq n\right\}$, where $\operatorname{deg}_{z_{i}}(f)$ is the degree of $f$ in $z_{i}$. If $\Psi \subset \mathbb{C}$ and $\Omega=\Psi^{n}$ then $\Omega$-stable polynomials will also be referred to as $\Psi$-stable. The fundamental classification problems that we address below are as follows.

Problem 1. Characterize all linear operators $T: \mathbb{K}_{\kappa}\left[z_{1}, \ldots, z_{n}\right] \rightarrow \mathbb{K}\left[z_{1}, \ldots, z_{n}\right]$ that preserve $\Omega$-stability for a given set $\Omega \subset \mathbb{C}^{n}$ and $\kappa \in \mathbb{N}^{n}$.

Problem 2. Characterize all linear operators $T: \mathbb{K}\left[z_{1}, \ldots, z_{n}\right] \rightarrow \mathbb{K}\left[z_{1}, \ldots, z_{n}\right]$ that preserve $\Omega$-stability, where $\Omega$ is a prescribed subset of $\mathbb{C}^{n}$.

For $n=1$ these problems were stated in this general form in [14, 17, see also [26] and [43, pp. 182-183]. Their natural multivariate analogs given above were formulated explicitly in 9, 10, thereby encompassing essentially all similar questions or variations thereof that have been much studied for more than a century. Note that for $n=1, \mathbb{K}=\mathbb{R}$ and $\Omega=\{z \in \mathbb{C}: \operatorname{Im}(z)>0\}$ Problems 12 amount to classifying linear operators that preserve the set of real polynomials with all real zeros. This question has a long and distinguished history going back to Laguerre and Pólya-Schur, see [10, 14] and the references therein. In particular, in [41] Pólya and Schur characterized all diagonal operators with this property (so-called multiplier sequences), which paved the way for numerous subsequent investigations 77, 9, 15, 16, 17, 19, 26, 32, 35, 40, 43, 50. However, it was not until very recently that full solutions to this question - and, more generally, to Problems 12 for $n=1$ and any open circular domain $\Omega$ - were obtained in [7].

In physics literature ([51], [53, §5]) one sometimes distinguishes between "soft" and "hard" theorems asserting the non-vanishing of partition functions in certain regions. This dichotomy stems from "soft-core" vs. "hard-core" pair interactions in lattice-gas models and does not refer to the level of difficulty in proving such theorems but rather to the fact that in some sense "soft" theorems are constraint-free while "hard" theorems involve constraints of various kinds, such as the maximum degree of a graph. By analogy with this terminology, one may say that results pertaining to Problem 1 are "hard" or "algebraic" (bounded degree) while those for Problem 2 are "soft" or "transcendental" (unbounded degree). For $n \geq 1$ several 
partial results of the latter type were established and appplied to various questions in e.g. combinatorics and statistical mechanics in the past two decades. In [33. Lieb and Sokal proved a general result [33. Proposition 2.2] pertaining to Problem 22 and more results of the same kind were recently obtained in [13, 57. Another contribution to this subject is Hinkkanen's Schur-Hadamard composition theorem 23. Progress on Problem 2 was recently made in [9] where a complete solution was given for finite order partial differential operators when $\Omega=\Psi^{n}$ and $\Psi$ is an open half-plane. Further contributions to this problem have been reported in [19].

The main results of this paper provide complete solutions to Problems 12 when $\Omega=\Omega_{1} \times \cdots \times \Omega_{n}$ and $\Omega_{i}, 1 \leq i \leq n$, are arbitrary open circular domains in $\mathbb{C}$. We also characterize all linear transformations preserving the class of Lee-Yang polynomials defined with respect to any such set $\Omega$. In particular, the classification theorems of [7] may now be viewed as special cases of the theorems below from which they follow by setting $n=1$. To achieve this we need several new ideas and results. For instance, we define the polarization of a linear operator to reduce the sufficiency part for arbitrary degrees in the algebraic characterization to the case of linear transformations acting on polynomials of degree at most one in each variable. For the necessity part in the transcendental characterization we generalize Szász' inequalities [55] to several variables. These are bounds on the coefficients of a stable polynomial that only depend on its first few non-zero coefficients.

The solutions to Problems 12 may be summarized as follows: (essentially all) linear operators preserving $n$-variate stable/Lee-Yang polynomials are induced by $2 n$-variate stable/Lee-Yang polynomials via appropriately defined symbol maps.

In a sequel [6] to this paper we build on our classification theorems to develop a self-contained theory of multivariate stable polynomials. We therefore take care to rely on as few auxiliary results as possible in the process. Combined with the present work, the theory and applications in [6] also yield a natural framework for dealing in a uniform manner with Lee-Yang type problems in statistical mechanics, combinatorics, and geometric function theory in one or several variables, thus contributing to Ruelle's quest [46] for an appropriate mathematical context encompassing the celebrated Lee-Yang theorem 31. (locating the zeros of the partition function of the ferromagnetic Ising model on the imaginary axis in the complex fugacity plane) and its many modern relatives [1, 3, 4, 5, 22, 27, 33, 37, 38, 45, 48, 51. This also illustrates Hinkkanen's observations about zeros of multivariate polynomials and their "so far unnoticed connections to various other concepts in mathematics" 23, which are further substantiated by recent applications to probability theory, matrix theory and combinatorics 8 , 11, 12, 13, 34, 44, 54, 57.

\section{Operator Symbols and $\Omega$-Stable Polynomials}

The general notion of $\Omega$-stability defined in the introduction extends classical univariate concepts such as Hurwitz or continuous-time stable polynomials and Schur or discrete-time stable polynomials (see, e.g., 35, 43]). These correspond to $n=1, \Omega=\{z \in \mathbb{C}: \operatorname{Re}(z) \geq 0\}$ and $n=1, \Omega=\{z \in \mathbb{C}:|z| \geq 1\}$, respectively.

Let now $n$ be an arbitrary positive integer. For $\theta \in[0,2 \pi)$ set

$$
\mathbb{H}_{\theta}=\left\{z \in \mathbb{C}: \operatorname{Im}\left(e^{i \theta} z\right)>0\right\} .
$$

We find it convenient to work with the upper half-plane

$$
H:=\mathbb{H}_{0}=\{z \in \mathbb{C}: \operatorname{Im}(z)>0\},
$$


therefore we will follow Levin's terminology [32] and refer to $H$-stable multivariate polynomials simply as stable polynomials (cf. [7, 8, 9, 11). A stable polynomial with all real coefficients is called real stable. Clearly, a univariate real polynomial is stable if and only if all its zeros are real. We denote the sets of stable, respectively real stable polynomials in $n$ variables by $\mathcal{H}_{n}(\mathbb{C})$ and $\mathcal{H}_{n}(\mathbb{R})$, respectively.

If $\Omega=\mathbb{H}_{\frac{\pi}{2}}=\{z \in \mathbb{C}: \operatorname{Re}(z)>0\}$ then $\Omega$-stable polynomials are called weakly Hurwitz stable. In [13] these are termed polynomials with the half-plane property.

1.1. Algebraic and Transcendental Symbols. The solutions to Problems 12 for circular domains make use of appropriately defined operator symbols that we proceed to describe. For simplicity, in this section we only state the main theorems for linear operators preserving $(H$-)stability.

Recall that $\mathbb{K}_{\kappa}\left[z_{1}, \ldots, z_{n}\right]$, where $\mathbb{K}=\mathbb{R}$ or $\mathbb{C}$ and $\kappa=\left(\kappa_{1}, \ldots, \kappa_{n}\right) \in \mathbb{N}^{n}$, is the $\mathbb{K}$-space of polynomials in variables $z_{1}, \ldots, z_{n}$ of degree at most $\kappa_{i}$ in $z_{i}, 1 \leq i \leq n$. If $\gamma=\left(\gamma_{1}, \ldots, \gamma_{n}\right) \in \mathbb{N}^{n}$ we define the algebraic symbol of a linear operator

$$
T: \mathbb{K}_{\kappa}\left[z_{1}, \ldots, z_{n}\right] \rightarrow \mathbb{K}_{\gamma}\left[z_{1}, \ldots, z_{n}\right]
$$

to be the polynomial $G_{T}(z, w) \in \mathbb{K}_{\gamma \oplus \kappa}\left[z_{1}, \ldots, z_{n}, w_{1}, \ldots, w_{n}\right]$ given by

$$
G_{T}(z, w)=T\left[(z+w)^{\kappa}\right]=\sum_{\alpha \leq \kappa}\left(\begin{array}{l}
\kappa \\
\alpha
\end{array}\right) T\left(z^{\alpha}\right) w^{\kappa-\alpha},
$$

where $\gamma \oplus \kappa=\left(\gamma_{1}, \ldots, \gamma_{n}, \kappa_{1}, \ldots, \kappa_{n}\right)$ while $\leq$ denotes the standard product partial order on $\mathbb{N}^{n}$ and we employ the usual multi-index notations $z=\left(z_{1}, \ldots, z_{n}\right), w=$ $\left(w_{1}, \ldots, w_{n}\right), z^{\alpha}=\prod_{i=1}^{n} z_{i}^{\alpha_{i}}, z w=\left(z_{1} w_{1}, \ldots, z_{n} w_{n}\right)$ and

$$
\left(\begin{array}{c}
\kappa \\
\alpha
\end{array}\right)=\left\{\begin{array}{l}
\prod_{j=1}^{n} \frac{\kappa_{j} !}{\left.\alpha_{j} ! \kappa_{j}-\alpha_{j}\right) !} \text { if } \alpha \leq \kappa, \\
0 \text { otherwise. }
\end{array}\right.
$$

Our first theorem provides an algebraic characterization of stability preservers on finite-dimensional complex polynomial spaces, which solves Problem 1 for $\mathbb{K}=\mathbb{C}$ and $\Omega=H$.

Theorem 1.1. Let $\kappa \in \mathbb{N}^{n}$ and $T: \mathbb{C}_{\kappa}\left[z_{1}, \ldots, z_{n}\right] \rightarrow \mathbb{C}\left[z_{1}, \ldots, z_{n}\right]$ be a linear operator. Then $T$ preserves stability if and only if either

(a) $T$ has range of dimension at most one and is of the form

$$
T(f)=\alpha(f) P,
$$

where $\alpha$ is a linear functional on $\mathbb{C}_{\kappa}\left[z_{1}, \ldots, z_{n}\right]$ and $P$ is a stable polynomial, or

(b) $G_{T}(z, w) \in \mathcal{H}_{2 n}(\mathbb{C})$.

Remark 1.1. Theorem 1.1 may also be stated in terms of other (algebraic) symbols of $T$ that are equivalent to the one defined above, for instance the polynomial

$$
T\left[(1-z w)^{\kappa}\right]=\sum_{\alpha \leq \kappa}(-1)^{\alpha}\left(\begin{array}{c}
\kappa \\
\alpha
\end{array}\right) T\left(z^{\alpha}\right) w^{\alpha}=(-1)^{\kappa} w^{\kappa} G_{T}\left(z,-w^{-1}\right) .
$$

Note that $T\left[(1-z w)^{\kappa}\right] \in \mathcal{H}_{2 n}(\mathbb{C}) \Leftrightarrow G_{T}(z, w) \in \mathcal{H}_{2 n}(\mathbb{C})$, see, e.g., Lemma 1.7 (3).

Definition 1.1. Two polynomials $f, g \in \mathbb{R}\left[z_{1}, \ldots, z_{n}\right]$ are in proper position, denoted $f \ll g$, if $g+$ if $\in \mathcal{H}_{n}(\mathbb{C})$. 
By the Hermite-Biehler theorem (see, e.g., [43, Theorem 6.3.4]) in the univariate case the relation $f \ll g$ is equivalent to saying that $f$ and $g$ are real-rooted (or identically zero), their zeros interlace and their Wronskian $W[f, g]:=f^{\prime} g-f g^{\prime}$ is non-positive on the whole of $\mathbb{R}$. We study further properties of this relation in $\$ 1.2$

The algebraic characterization of real stability preservers on finite-dimensional polynomial spaces - which solves Problem 1 for $\mathbb{K}=\mathbb{R}$ and $\Omega=H$ - is as follows.

Theorem 1.2. Let $\kappa \in \mathbb{N}^{n}$ and $T: \mathbb{R}_{\kappa}\left[z_{1}, \ldots, z_{n}\right] \rightarrow \mathbb{R}\left[z_{1}, \ldots, z_{n}\right]$ be a linear operator. Then $T$ preserves real stability if and only if either

(a) $T$ has range of dimension no greater than two and is of the form

$$
T(f)=\alpha(f) P+\beta(f) Q,
$$

where $\alpha, \beta: \mathbb{R}_{\kappa}\left[z_{1}, \ldots, z_{n}\right] \rightarrow \mathbb{R}$ are linear functionals and $P, Q$ are real stable polynomials such that $P \ll Q$, or

(b) $G_{T}(z, w) \in \mathcal{H}_{2 n}(\mathbb{R})$, or

(c) $G_{T}(z,-w) \in \mathcal{H}_{2 n}(\mathbb{R})$.

If $T: \mathbb{K}\left[z_{1}, \ldots, z_{n}\right] \rightarrow \mathbb{K}\left[z_{1}, \ldots, z_{n}\right]$, where $\mathbb{K}=\mathbb{R}$ or $\mathbb{C}$, is a linear operator we define its transcendental symbol, $\bar{G}_{T}(z, w)$, to be the formal power series in $w_{1}, \ldots, w_{n}$ with polynomial coefficients in $\mathbb{K}\left[z_{1}, \ldots, z_{n}\right]$ given by

$$
\bar{G}_{T}(z, w)=\sum_{\alpha \in \mathbb{N}^{n}}(-1)^{\alpha} T\left(z^{\alpha}\right) \frac{w^{\alpha}}{\alpha !}
$$

where $\alpha !=\alpha_{1} ! \cdots \alpha_{n} !$. By abuse of notation we write $\bar{G}_{T}(z, w)=T\left[e^{-z \cdot w}\right]$, where $z \cdot w=z_{1} w_{1}+\ldots+z_{n} w_{n}$.

Let us define the complex Laguerre-Pólya class $\overline{\mathcal{H}}_{n}(\mathbb{C})$ as the class of entire functions in $n$ variables that are limits, uniformly on compact sets, of polynomials in $\mathcal{H}_{n}(\mathbb{C})$, see, e.g., [32, Chap. IX]. The usual Laguerre-Pólya class $\overline{\mathcal{H}}_{n}(\mathbb{R})$ consists of all functions in $\overline{\mathcal{H}}_{n}(\mathbb{C})$ with real coefficients.

Our next theorem provides a transcendental characterization of stability preservers on infinite-dimensional complex polynomial spaces, thus solving Problem 2 for $\mathbb{K}=\mathbb{C}$ and $\Omega=H$.

Theorem 1.3. Let $T: \mathbb{C}\left[z_{1}, \ldots, z_{n}\right] \rightarrow \mathbb{C}\left[z_{1}, \ldots, z_{n}\right]$ be a linear operator. Then $T$ preserves stability if and only if either

(a) $T$ has range of dimension at most one and is of the form

$$
T(f)=\alpha(f) P,
$$

where $\alpha$ is a linear functional on $\mathbb{C}\left[z_{1}, \ldots, z_{n}\right]$ and $P$ is a stable polynomial, or

(b) $\bar{G}_{T}(z, w) \in \overline{\mathcal{H}}_{2 n}(\mathbb{C})$.

Remark 1.2. From Theorem 1.3 one can easily deduce a characterization of linear operators preserving $\Omega$-stability for any open half-plane $\Omega$. For instance, the analog of Theorem 1.3 (b) for the open right half-plane $\mathbb{H}_{\frac{\pi}{2}}$ is that the transcendental symbol of $T$ with respect to $\mathbb{H}_{\frac{\pi}{2}}$, i.e., the formal power series

$$
T\left[e^{z \cdot w}\right]:=\sum_{\alpha \in \mathbb{N}^{n}} T\left(z^{\alpha}\right) \frac{w^{\alpha}}{\alpha !},
$$

defines an entire function which is the limit, uniformly on compact sets, of weakly Hurwitz stable polynomials. 
The analog of Theorem 1.3 for real stability presevers - which solves Problem 2 for $\mathbb{K}=\mathbb{R}$ and $\Omega=H-$ is as follows.

Theorem 1.4. Let $T: \mathbb{R}\left[z_{1}, \ldots, z_{n}\right] \rightarrow \mathbb{R}\left[z_{1}, \ldots, z_{n}\right]$ be a linear operator. Then $T$ preserves real stability if and only if either

(a) $T$ has range of dimension no greater than two and is of the form

$$
T(f)=\alpha(f) P+\beta(f) Q
$$

where $\alpha, \beta: \mathbb{R}\left[z_{1}, \ldots, z_{n}\right] \rightarrow \mathbb{R}$ are linear functionals and $P, Q$ are real stable polynomials such that $P \ll Q$, or

(b) $\bar{G}_{T}(z, w) \in \overline{\mathcal{H}}_{2 n}(\mathbb{R})$, or

(c) $\bar{G}_{T}(z,-w) \in \overline{\mathcal{H}}_{2 n}(\mathbb{R})$.

1.2. Fundamental Properties of Stable Polynomials. In this section we first review some basic facts and then prove several results on (real) stable polynomials needed later on. We start with an immediate consequence of the definitions (cf. [9, Lemma 2.1]).

Lemma 1.5. Let $f \in \mathbb{K}\left[z_{1}, \ldots, z_{n}\right]$, where $\mathbb{K}=\mathbb{R}$ or $\mathbb{C}$. Then $f \in \mathcal{H}_{n}(\mathbb{K})$ if and only if $f(\lambda t+\alpha) \in \mathcal{H}_{1}(\mathbb{K})$ for all $\lambda \in \mathbb{R}_{+}^{n}$ and $\alpha \in \mathbb{R}^{n}$.

The following theorem is a multivariate version of Hurwitz' theorem on the "continuity of zeros", see, e.g., [13, Footnote 3, p. 96] for a proof.

Theorem 1.6 (Hurwitz' theorem). Let $D$ be a domain (open connected set) in $\mathbb{C}^{n}$ and suppose $\left\{f_{k}\right\}_{k=1}^{\infty}$ is a sequence of non-vanishing analytic functions on $D$ that converge to $f$ uniformly on compact subsets of $D$. Then $f$ is either non-vanishing on $D$ or else identically zero.

We next list some of the closure properties for (real) stable polynomials. The first property in Lemma 1.7 below is deduced by applying Theorem 1.6 with $D=$ $\{z \in \mathbb{C}: \operatorname{Im}(z)>0\}^{n}$ and $f_{k}\left(z_{1}, \ldots, z_{n}\right)=f\left(z_{1}, \ldots, z_{i-1}, \mu+z_{i} / k, z_{i+1}, \ldots, z_{n}\right)$, $k \in \mathbb{N}$, while the remaining properties are easy consequences of the definitions and Lemma 1.5.

Lemma 1.7. Let $\mathbb{K}=\mathbb{R}$ or $\mathbb{C}$ and $f \in \mathcal{H}_{n}(\mathbb{K})$ be of degree $d_{j}$ in $z_{j}, 1 \leq j \leq n$. Then for any $1 \leq i \leq n$ one has:

(1) $f\left(z_{1}, \ldots, z_{i-1}, \mu, z_{i+1}, \ldots, z_{n}\right) \in \mathcal{H}_{n-1}(\mathbb{K}) \cup\{0\}$ for $\mu \in \mathbb{R}$;

(2) $f\left(z_{1}, \ldots, z_{i-1}, \lambda z_{i}, z_{i+1}, \ldots, z_{n}\right) \in \mathcal{H}_{n}(\mathbb{K})$ for $\lambda>0$;

(3) $z_{i}^{d_{i}} f\left(z_{1}, \ldots, z_{i-1},-z_{i}^{-1}, z_{i+1}, \ldots, z_{n}\right) \in \mathcal{H}_{n}(\mathbb{K})$;

(4) $f\left(z_{1}, \ldots, z_{i-1}, z_{j}, z_{i+1}, \ldots, z_{n}\right) \in \mathcal{H}_{n-1}(\mathbb{K})$ for $1 \leq j \neq i \leq n$.

We will now establish a series of results involving the notion of proper position introduced in Definition [1.1, compare with [9, §2], [13, Theorem 3.2]. In particular, the next lemma provides a multivariate analog of the classical Hermite-Biehler theorem showing that proper position is a natural higher dimensional counterpart of interlacing.

Lemma 1.8. Let $f, g \in \mathbb{R}\left[z_{1}, \ldots, z_{n}\right] \backslash\{0\}$, set $h=f+i g$ and suppose that $f$ and $g$ are not constant multiples of each other. The following are equivalent:

(1) $h \in \mathcal{H}_{n}(\mathbb{C})$, that is, $g \ll f$;

(2) $|h(z)|>|h(\bar{z})|$ for all $z=\left(z_{1}, \ldots, z_{n}\right) \in \mathbb{C}^{n}$ with $\operatorname{Im}\left(z_{j}\right)>0,1 \leq j \leq n$, where $\bar{z}=\left(\bar{z}_{1}, \ldots, \bar{z}_{n}\right)$; 
(3) $f+z_{n+1} g \in \mathcal{H}_{n+1}(\mathbb{R})$;

(4) $f, g \in \mathcal{H}_{n}(\mathbb{R})$ and

$$
\operatorname{Im}\left(\frac{f(z)}{g(z)}\right) \geq 0
$$

whenever $z=\left(z_{1}, \ldots, z_{n}\right) \in \mathbb{C}^{n}$ with $\operatorname{Im}\left(z_{j}\right)>0,1 \leq j \leq n$;

(5) $g(\lambda t+\alpha) \ll f(\lambda t+\alpha)$ for all $\lambda \in \mathbb{R}_{+}^{n}$ and $\alpha \in \mathbb{R}^{n}$.

Proof. The equivalences $(1) \Leftrightarrow(5)$ and $(3) \Leftrightarrow(4)$ are immediate from the definitions and Lemma 1.5. It is also clear that $(3) \Rightarrow(1)$ and $(2) \Rightarrow(1)$. We proceed to prove the remaining implications, namely $(1) \Rightarrow(3)$ and $(1) \Rightarrow(2)$.

Suppose that (1) holds, i.e., $h \in \mathcal{H}_{n}(\mathbb{C})$, and fix $w=\alpha+i \beta \in\{\zeta \in \mathbb{C}: \operatorname{Im}(\zeta)>$ $0\}^{n}$ with $\alpha, \beta \in \mathbb{R}^{n}$. The polynomial $p(t)=h(\alpha+t \beta)$ is stable so we may write it as

$$
p(t)=C \prod_{j=1}^{d}\left(t-\zeta_{j}\right),
$$

where $C \in \mathbb{C}$ and $\operatorname{Im}\left(\zeta_{j}\right) \leq 0,1 \leq j \leq d$. Note that for all $j$ one has $\left|i-\zeta_{j}\right| \geq\left|-i-\zeta_{j}\right|$ with equality only if $\zeta_{j} \in \mathbb{R}$. Hence

$$
|f(w)+i g(w)|=|h(w)| \geq|h(\bar{w})|=|\overline{h(\bar{w})}|=|f(w)-i g(w)| .
$$

To prove (3) we need to show that the univariate polynomial $q\left(z_{n+1}\right)=f(w)+$ $z_{n+1} g(w)$ is stable. If $g(w)=0$ then $q\left(z_{n+1}\right)$ is a non-zero constant so it is stable. Therefore we may assume that $g(w) \neq 0$. By the above we have $|f(w) / g(w)+i| \geq$ $|f(w) / g(w)-i|$, which implies that $\operatorname{Im}(f(w) / g(w)) \geq 0$. Now if $q\left(z_{n+1}\right)=0$ then $f(w) / g(w)+z_{n+1}=0$ and thus $\operatorname{Im}\left(z_{n+1}\right) \leq 0$. It follows that $q \in \mathcal{H}_{1}(\mathbb{C})$ and since $w \in\{\zeta \in \mathbb{C}: \operatorname{Im}(\zeta)>0\}^{n}$ was arbitrarily fixed we deduce that $f+z_{n+1} g \in \mathcal{H}_{n+1}(\mathbb{C})$ hence $f+z_{n+1} g \in \mathcal{H}_{n+1}(\mathbb{R})$, which confirms (3).

Finally, to show that $(1) \Rightarrow(2)$ note that by the above it is actually enough to prove that $(3) \Rightarrow(2)$. Now if (3) holds then letting $z_{n+1}=i$ we get in particular that $h \in \mathcal{H}_{n}(\mathbb{C})$ and therefore $|f(w)+i g(w)| \geq|f(w)-i g(w)|$ for all $w \in\{\zeta \in \mathbb{C}:$ $\operatorname{Im}(\zeta)>0\}^{n}$. It remains to show that we cannot have $|f(w)+i g(w)|=|f(w)-i g(w)|$ for such $w$. Supposing the contrary it follows from the above arguments that for some $\alpha \in \mathbb{R}^{n}$ and $\beta \in \mathbb{R}_{+}^{n}$ all the zeros $\zeta_{j}, 1 \leq j \leq d$, of the polynomial $p(t)=f(\alpha+t \beta)+i g(\alpha+t \beta)$ are real. Since $f(\alpha+t \beta)$ and $g(\alpha+t \beta)$ have real coefficients this can occur only if these polynomials are constant multiples of each other, say $f(\alpha+t \beta)+a g(\alpha+t \beta)=0$ for some $a \in \mathbb{R}$. By setting the variable $z_{n+1}=a$ we see that $f(z)+a g(z)$ is either a stable polynomial or identically zero. Since it vanishes for $z=\alpha+i \beta$ it must be identically zero, which contradicts the assumption that $f$ and $g$ are not constant multiples of each other. We conclude that $(3) \Rightarrow(2)$, which completes the proof of the lemma.

Remark 1.3. The equivalence $(3) \Leftrightarrow(4)$ in Lemma 1.8 extends in obvious fashion to $\mathbb{H}_{\theta}$-stable polynomials with complex coefficients: if $f, g \in \mathbb{C}\left[z_{1}, \ldots, z_{n}\right] \backslash\{0\}$ then $f+z_{n+1} g$ is $\mathbb{H}_{\theta}$-stable (in $n+1$ variables) if and only if

$$
\operatorname{Im}\left(\frac{e^{i \theta} f(z)}{g(z)}\right) \geq 0, \quad z=\left(z_{1}, \ldots, z_{n}\right) \in \mathbb{H}_{\theta}^{n} .
$$


Note in particular that for the open right-half plane $\mathbb{H}_{\frac{\pi}{2}}$ the above relation reads

$$
\operatorname{Re}\left(\frac{f(z)}{g(z)}\right) \geq 0, \quad \operatorname{Re}\left(z_{j}\right)>0,1 \leq j \leq n .
$$

Definition 1.2. For $g, f \in \mathbb{R}\left[z_{1}, \ldots, z_{n}\right]$ and $1 \leq j \leq n$ define the $j$-th Wronskian of $g, f$ as

$$
W_{j}[g, f]=\frac{\partial g}{\partial z_{j}} \cdot f-g \cdot \frac{\partial f}{\partial z_{j}} .
$$

The following multivariate analog of the Hermite-Kakeya-Obreschkoff theorem (see, e.g., [43, Theorem 6.3.8] for the classical univariate version) was first proved in [9. Below we give an alternative shorter proof.

Theorem 1.9. Let $f, g \in \mathbb{R}\left[z_{1}, \ldots, z_{n}\right]$. All non-zero polynomials in the space

$$
\{\alpha f+\beta g: \alpha, \beta \in \mathbb{R}\}
$$

are stable if and only if either $f=g \equiv 0, f \ll g$ or $g \ll f$. Moreover, if $g \ll f$ then $W_{j}[g, f](x) \leq 0$ for all $x \in \mathbb{R}^{n}$ and $1 \leq j \leq n$, and if $f \ll g$ then $W_{j}[g, f](x) \geq 0$ for all $x \in \mathbb{R}^{n}$ and $1 \leq j \leq n$.

Proof. If $f$ and $g$ are linearly dependent then both conditions say that $f$ and $g$ are real stable or zero and then the Wronskians are all zero. Hence we may assume that $f, g \in \mathbb{R}\left[z_{1}, \ldots, z_{n}\right] \backslash\{0\}$ are not constant multiples of each other.

The "if" direction follows from Lemma $1.8(1) \Rightarrow(3)$ and Lemma 1.7 (1).

For the converse assume that all non-zero polynomials in the space $\{\alpha f+\beta g$ : $\alpha, \beta \in \mathbb{R}\}$ are stable and that there exist $z, w \in\{\zeta \in \mathbb{C}: \operatorname{Im}(\zeta)>0\}^{n}$ for which

$$
\operatorname{Im}\left(\frac{f(z)}{g(z)}\right)>0 \quad \text { and } \quad \operatorname{Im}\left(\frac{f(w)}{g(w)}\right)<0
$$

By connectivity there is a number $v \in\{\zeta \in \mathbb{C}: \operatorname{Im}(\zeta)>0\}^{n}$ for which $f(v) / g(v)=$ $a \in \mathbb{R} \backslash\{0\}$. It follows that $f-a g$ is identically zero which we assumed was not the case. Hence $\operatorname{Im}(f(z) / g(z))$ has constant sign for $z \in\{\zeta \in \mathbb{C}: \operatorname{Im}(\zeta)>0\}^{n}$ and we conclude, by Lemma $1.8(4) \Rightarrow(1)$, that either $f \ll g$ or $g \ll f$.

Suppose now that $g \ll f$, where we may assume that $g$ is not identically zero. Let $x \in \mathbb{R}^{n}$ be such that $g(x) \neq 0$ and consider the rational function $q(t)=$ $f\left(x+e_{j} t\right) / g\left(x+e_{j} t\right)$, where $e_{j}$ is the $j$-th standard basis vector of $\mathbb{R}^{n}$. Hence $q(t)$ is analytic at the origin with a first order Taylor expansion given by

$$
q(t)=\frac{f(x)}{g(x)}-\frac{W_{j}[g, f](x)}{g^{2}(x)} t+O\left(t^{2}\right)
$$

From Lemma 1.8 and the fact that $\operatorname{Im}(f(x) / g(x))=0$ we then get $W_{j}[g, f](x) \leq 0$. Since the set of all $x \in \mathbb{R}^{n}$ for which $g(x) \neq 0$ is dense in $\mathbb{R}^{n}$ the theorem follows.

Corollary 1.10. If $f, g \in \mathbb{R}\left[z_{1}, \ldots, z_{n}\right] \backslash\{0\}$ are real stable polynomials such that $f \ll g$ and $g \ll f$ then $f=\alpha g$ for some $\alpha \in \mathbb{R}$.

Proof. If $f, g \in \mathbb{R}\left[z_{1}, \ldots, z_{n}\right] \backslash\{0\}$ are real stable polynomials such that $f \ll g$ and $g \ll f$ then $f \ll g$ and $f \ll-g$. Hence, by Theorem 1.9

$$
\frac{\partial}{\partial z_{j}}\left(\frac{g}{f}\right)(x)=\frac{W_{j}[g, f](x)}{f(x)^{2}}=0
$$

for all $1 \leq j \leq n$ and $x \in \mathbb{R}^{n}$ for which $f(x) \neq 0$. Thus $g / f$ is a real constant. 


\section{Hard-Core/Algebraic Classification: Sufficiency}

2.1. Multi-affine Polynomials and the Lieb-Sokal Lemma. A polynomial $f \in \mathbb{C}\left[z_{1}, \ldots, z_{n}\right]$ is multi-affine if all monomials in its Taylor expansion are squarefree, i.e., if $f$ can be written as

$$
f(z)=\sum_{S \subseteq[n]} a(S) z^{S}, \text { where } z^{S}:=\prod_{i \in S} z_{i}, \quad a(S) \in \mathbb{C}, \quad[n]=\{1, \ldots, n\} .
$$

Hence $f \in \mathbb{C}\left[z_{1}, \ldots, z_{n}\right]$ is multi-affine if and only if $f \in \mathbb{C}_{\left(1^{n}\right)}\left[z_{1}, \ldots, z_{n}\right]$, where $\left(1^{n}\right)=(1, \ldots, 1) \in \mathbb{N}^{n}$.

The following lemma (in the case $n=1$ ) is due to Lieb and Sokal [33, Lemma 2.3]. For completeness we provide here a short proof essentially based on the same idea.

Lemma 2.1 (Lieb-Sokal). Let $P(z)+w Q(z) \in \mathbb{C}\left[z_{1}, \ldots, z_{n}, w\right]$ be stable. If the degree in the variable $z_{j}$ is at most one then the polynomial

$$
P(z)-\frac{\partial Q(z)}{\partial z_{j}}
$$

is either identically zero or stable.

Proof. We may assume that $Q(z)$ is not identically equal to zero and that $j=1$. Since $Q(z)$ is stable and $\operatorname{Im}(w)>0 \Leftrightarrow \operatorname{Im}\left(-w^{-1}\right)>0$ the polynomial

$$
w Q(z)-\frac{\partial Q(z)}{\partial z_{1}}=w Q\left(z_{1}-w^{-1}, z_{2}, \ldots, z_{n}\right)
$$

is stable. Hence, by Remark 1.3 (with $\theta=0$ ) one has

$$
\operatorname{Im}\left(\frac{P(z)-\partial Q(z) / \partial z_{1}}{Q(z)}\right)=\operatorname{Im}\left(\frac{P(z)}{Q(z)}\right)+\operatorname{Im}\left(\frac{-\partial Q(z) / \partial z_{1}}{Q(z)}\right) \geq 0
$$

for all $z \in\{\zeta \in \mathbb{C}: \operatorname{Im}(\zeta)>0\}^{n}$, so by Remark 1.3 again the polynomial

$$
P(z)-\frac{\partial Q(z)}{\partial z_{1}}+w Q(z)
$$

is stable. In light of Lemma 1.7(1), this implies in particular the conclusion of the lemma (letting $w=0$ ).

We can now settle the sufficiency part of Theorem 1.1 in the case of multi-affine polynomials, i.e., for $\kappa=(1, \ldots, 1)$.

Lemma 2.2. Let $T: \mathbb{C}_{\left(1^{n}\right)}\left[z_{1}, \ldots, z_{n}\right] \rightarrow \mathbb{C}\left[z_{1}, \ldots, z_{n}\right]$ be a linear operator such that

$$
G_{T}(z, w):=T\left[(z+w)^{[n]}\right]=\sum_{S \subseteq[n]} T\left[z^{S}\right] w^{[n] \backslash S}
$$

is stable, where $z=\left(z_{1}, \ldots, z_{m}\right)$ and $w=\left(w_{1}, \ldots, w_{n}\right)$. Then $T$ preserves stability.

Proof. Let $T$ be as in the lemma. Since $\operatorname{Im}\left(w_{j}\right)>0 \Leftrightarrow \operatorname{Im}\left(-w_{j}^{-1}\right)>0$ we have that

$$
w^{[n]} G_{T}\left(z,-w^{-1}\right) \in \mathcal{H}_{2 n}(\mathbb{C}) \Longleftrightarrow G_{T}(z, w) \in \mathcal{H}_{2 n}(\mathbb{C}) .
$$

Therefore, if $f \in \mathbb{C}\left[v_{1}, \ldots, v_{n}\right]$ is stable and multi-affine we deduce that

$$
w^{[n]} G_{T}\left(z,-w^{-1}\right) f(v)=\sum_{S \subseteq[n]} T\left[z^{S}\right](-w)^{S} f(v) \in \mathcal{H}_{3 n}(\mathbb{C})
$$


where $v=\left(v_{1}, \ldots, v_{n}\right)$. By repeated use of Lieb-Sokal's Lemma 2.1 we then get

$$
\sum_{S \subseteq[n]} T\left[z^{S}\right] f^{(S)}(v) \in \mathcal{H}_{2 n}(\mathbb{C}) \cup\{0\} .
$$

Letting $\{\zeta \in \mathbb{C}: \operatorname{Im}(\zeta)>0\}^{n} \ni v \rightarrow 0$ and invoking Hurwitz' Theorem 1.6 we obtain

$$
T(f)=\sum_{S \subseteq[n]} T\left[z^{S}\right] f^{(S)}(0) \in \mathcal{H}_{n}(\mathbb{C}) \cup\{0\},
$$

which proves the lemma.

2.2. Decoupling Schemes: Polarizations of Polynomials and Operators. For $\kappa=\left(\kappa_{1}, \ldots, \kappa_{n}\right) \in \mathbb{N}^{n}$ let $\mathbb{C}_{M A}^{\kappa}$ be the space of multi-affine polynomials in the variables $\left\{z_{i j}: 1 \leq i \leq n, 1 \leq j \leq \kappa_{i}\right\}$. Define a (linear) polarization operator

$$
\Pi_{\kappa}^{\uparrow}: \mathbb{C}_{\kappa}\left[z_{1}, \ldots, z_{n}\right] \rightarrow \mathbb{C}_{M A}^{\kappa}
$$

that associates to each $f \in \mathbb{C}_{\kappa}\left[z_{1}, \ldots, z_{n}\right]$ the unique polynomial $\Pi_{\kappa}^{\uparrow}(f) \in \mathbb{C}_{M A}^{\kappa}$ such that

(a) for any $1 \leq i \leq n$ the polynomial $\Pi_{\kappa}^{\uparrow}(f)$ is symmetric in $\left\{z_{i j}: 1 \leq j \leq \kappa_{i}\right\}$;

(b) if we let $z_{i j}=z_{i}$ for all $1 \leq i \leq n$ and $1 \leq j \leq \kappa_{i}$ in $\Pi_{\kappa}^{\uparrow}(f)$ we recover $f$.

In other words, if $\alpha \leq \kappa$ then

$$
\Pi_{\kappa}^{\uparrow}\left(z^{\alpha}\right)=\left(\begin{array}{c}
\kappa \\
\alpha
\end{array}\right)^{-1} E_{\alpha_{1}}\left(z_{11}, \ldots, z_{1 \kappa_{1}}\right) \cdots E_{\alpha_{n}}\left(z_{n 1}, \ldots, z_{n \kappa_{n}}\right),
$$

where $E_{i}\left(x_{1}, \ldots, x_{m}\right)$ is the $i$-th elementary symmetric polynomial in the variables $x_{1}, \ldots, x_{m}$, that is,

$$
E_{i}\left(x_{1}, \ldots, x_{m}\right)=\sum_{S \subseteq[m],|S|=i} x^{S}, \quad 0 \leq i \leq m .
$$

Dually we define a (linear) projection operator

$$
\Pi_{\kappa}^{\downarrow}: \mathbb{C}_{M A}^{\kappa} \rightarrow \mathbb{C}_{\kappa}\left[z_{1}, \ldots, z_{n}\right]
$$

by letting $z_{i j} \mapsto z_{i}$ and extending linearly. Note that by (b) above $\Pi_{\kappa}^{\downarrow} \circ \Pi_{\kappa}^{\uparrow}$ is the identity operator on $\mathbb{C}_{\kappa}\left[z_{1}, \ldots, z_{n}\right]$ while $\Pi_{\kappa}^{\uparrow} \circ \Pi_{\kappa}^{\downarrow}$ is the operator on $\mathbb{C}_{M A}^{\kappa}$ that for each $1 \leq i \leq n$ symmetrizes all the variables in $\left\{z_{i j}: 1 \leq j \leq \kappa_{i}\right\}$.

Remark 2.1. In physics literature polarization and projection operators as above have mostly been used for univariate polynomials and are often referred to as "decoupling procedures" [3, 31. Such procedures combined with Pólya type results [39, Hilfssatz II] were employed by Lee and Yang in their original proof of the "circle theorem" [31, Appendix II] (see also Kac's comments in [40, pp. 424-426] and [6, $\S 9])$. Multivariate polarization notions similar to those defined above are used in the theory of hyperbolic polynomials and partial differential equations [21, 25, 29.

Let $T: \mathbb{C}_{\kappa}\left[z_{1}, \ldots, z_{n}\right] \rightarrow \mathbb{C}_{\gamma}\left[z_{1}, \ldots, z_{n}\right]$ be a linear operator. The polarization of $T$ is defined as the linear operator $\Pi(T): \mathbb{C}_{M A}^{\kappa} \rightarrow \mathbb{C}_{M A}^{\gamma}$ given by

$$
\Pi(T)=\Pi_{\gamma}^{\uparrow} \circ T \circ \Pi_{\kappa}^{\downarrow},
$$

and conversely we have

$$
T=\Pi_{\gamma}^{\downarrow} \circ \Pi(T) \circ \Pi_{\kappa}^{\uparrow}
$$


It is immediate from Lemma 1.7 (4) that the projection operator $\Pi_{\kappa}^{\downarrow}$ preserves stability, but the remarkable fact is that so does $\Pi_{\kappa}^{\uparrow}$. This is essentially the famous Grace-Walsh-Szegö coincidence theorem [20, 56, 58, albeit in a disguised form. For completeness let us state this theorem.

Theorem 2.3 (Grace-Walsh-Szegö). Let $f$ be a symmetric multi-affine polynomial in $n$ complex variables, let $C$ be an open or closed circular domain, and let $\xi_{1}, \ldots, \xi_{n}$ be points in $C$. Suppose further that either the total degree of $f$ equals $n$ or that $C$ is convex (or both). Then there exists at least one point $\xi \in C$ such that

$$
f\left(\xi_{1}, \ldots, \xi_{n}\right)=f(\xi, \ldots, \xi) .
$$

We give a new and self-contained proof of Theorem [2.3 in [6, §2].

Proposition 2.4. Let $f \in \mathbb{C}_{\kappa}\left[z_{1}, \ldots, z_{n}\right]$. Then $f$ is stable if and only if $\Pi_{\kappa}^{\uparrow}(f)$ is stable.

Proof. The "if" direction follows from the fact that $\Pi_{\kappa}^{\downarrow}$ preserves stability. For the other direction it suffices to prove the theorem for univariate polynomials since we may polarize the variables one at a time (when the other variables are fixed in the upper half-plane). Suppose therefore that $f(z)=\sum_{k=0}^{d} a_{k} z^{k}$ is a univariate stable polynomial. Then the polarization of $f$ is given by

$$
F\left(z_{1}, \ldots, z_{n}\right)=\sum_{k=0}^{d} a_{k}\left(\begin{array}{l}
d \\
k
\end{array}\right)^{-1} E_{k}\left(z_{1}, \ldots, z_{d}\right) .
$$

If $F\left(\zeta_{1}, \ldots, \zeta_{n}\right)=0$ for some $\left(\zeta_{1}, \ldots, \zeta_{n}\right) \in H^{n}$ then by the Grace-Walsh-Szegö theorem (Theorem 2.3) there exists $\zeta \in H$ such that $f(\zeta)=F(\zeta, \ldots, \zeta)=0$, contradicting the stability of $f$.

Lemma 2.5. Let $T: \mathbb{C}_{\kappa}\left[z_{1}, \ldots, z_{n}\right] \rightarrow \mathbb{C}_{\gamma}\left[z_{1}, \ldots, z_{n}\right]$ be a linear operator. Then the symbol of the polarization of $T$ is the polarization of the symbol of $T$, that is,

$$
G_{\Pi(T)}=\Pi_{\gamma \oplus \kappa}^{\uparrow}\left(G_{T}\right),
$$

where $\Pi_{\gamma \oplus \kappa}^{\uparrow}: \mathbb{C}_{\gamma \oplus \kappa}\left[z_{1}, \ldots, z_{n}, w_{1}, \ldots, w_{n}\right] \rightarrow \mathbb{C}_{M A}^{\gamma \oplus \kappa}$.

Proof. We have that

$$
\Pi_{\kappa}^{\downarrow z}\left[\prod_{i=1}^{n} \prod_{j=1}^{\kappa_{i}}\left(z_{i j}+w_{i j}\right)\right]=\Pi_{\kappa}^{\downarrow z}\left[\left(\Pi_{\kappa}^{\uparrow z} \circ \Pi_{\kappa}^{\uparrow w}\right)\left[(z+w)^{\kappa}\right]\right],
$$

where the upper indices $z$ and $w$ indicate that the corresponding operators act only on the $z$-variables and $w$-variables, respectively. Hence the symbol of the polarized operator may be written as

$$
\begin{aligned}
G_{\Pi(T)} & =\left(\Pi_{\gamma}^{\uparrow z} \circ T \circ \Pi_{\kappa}^{\downarrow z}\right)\left[\prod_{i=1}^{n} \prod_{j=1}^{\kappa_{i}}\left(z_{i j}+w_{i j}\right)\right] \\
& =\left(\Pi_{\gamma}^{\uparrow z} \circ T \circ \Pi_{\kappa}^{\downarrow z}\right)\left[\left(\Pi_{\kappa}^{\uparrow z} \circ \Pi_{\kappa}^{\uparrow w}\right)\left[(z+w)^{\kappa}\right]\right] .
\end{aligned}
$$

Using the fact that $\Pi_{\kappa}^{\downarrow z} \circ \Pi_{\kappa}^{\uparrow z}$ is the identity operator and that $T$ and $\Pi_{\kappa}^{\uparrow w}$ commute (since $T$ only acts on the $z$-variables) we get

$$
G_{\Pi(T)}=\left(\Pi_{\gamma}^{\uparrow z} \circ \Pi_{\kappa}^{\uparrow w} \circ T\right)\left[(z+w)^{\kappa}\right]=\Pi_{\gamma \oplus \kappa}^{\uparrow}\left(G_{T}\right),
$$


as desired.

Proof of Sufficiency in Theorem 1.1. If $G_{T}$ is stable then so is $G_{\Pi(T)}$ by Lemma2.5 and Proposition 2.4 From Lemma 2.2 we deduce that $\Pi(T)$ preserves stability, and then by equation (2.2) so does $T$ since in view of Lemma 1.7 and Proposition 2.4 both operators $\Pi_{\gamma}^{\downarrow}$ and $\Pi_{\kappa}^{\uparrow}$ preserve stability.

\section{Algebraic Classification: Necessity in the Complex Case}

We will need the following simple lemma which makes it clear that there are plenty of stable polynomials.

Lemma 3.1. Let $f \in \mathbb{C}_{\kappa}\left[z_{1}, \ldots, z_{n}\right]$, where $\kappa=\left(\kappa_{1}, \ldots, \kappa_{n}\right) \in \mathbb{N}^{n}$, and $W=$ $\left(W_{1}, \ldots, W_{n}\right) \in\{\zeta \in \mathbb{C}: \operatorname{Im}(\zeta)>0\}^{n}$. Then for all $\epsilon>0$ sufficiently small one has

$(z+W)^{\kappa}+\epsilon f(z) \in \mathcal{H}_{n}(\mathbb{C})$, where $(z+W)^{\kappa}=\left(z_{1}+W_{1}\right)^{\kappa_{1}} \cdots\left(z_{n}+W_{n}\right)^{\kappa_{n}}$.

Proof. Set $Y=\left(\operatorname{Im}\left(W_{1}\right), \ldots, \operatorname{Im}\left(W_{n}\right)\right) \in \mathbb{R}_{+}^{n}$. For $\alpha \leq \kappa$ we then have

$$
\left|\frac{(z+W)^{\alpha}}{(z+W)^{\kappa}}\right| \leq Y^{\alpha-\kappa} \text { if } z \in\{\zeta \in \mathbb{C}: \operatorname{Im}(\zeta) \geq 0\}^{n} .
$$

Expanding $f$ in powers of $z+W$ we see that there exists $\epsilon_{0}>0$ such that

$$
\frac{|f(z)|}{\left|(z+W)^{\kappa}\right|}<\frac{1}{\epsilon_{0}} \text { if } z \in\{\zeta \in \mathbb{C}: \operatorname{Im}(\zeta) \geq 0\}^{n} .
$$

Hence $\left|(z+W)^{\kappa}+\epsilon f(z)\right| \geq\left|(z+W)^{\kappa}\right|-\epsilon|f(z)|>0$ for all $z \in\{\zeta \in \mathbb{C}: \operatorname{Im}(\zeta) \geq 0\}^{n}$ and $\epsilon \in\left(0, \epsilon_{0}\right)$, so that in particular $(z+W)^{\kappa}+\epsilon f(z) \in \mathcal{H}_{n}(\mathbb{C})$ for all such $\epsilon$.

Remark 3.1. From the proof of Lemma 3.1 it follows that the topological dimension of the set $\mathcal{H}_{n}(\mathbb{C}) \cap \mathbb{C}_{\kappa}\left[z_{1}, \ldots, z_{n}\right]$ (of all stable polynomials in $n$ variables of degree at most $\kappa$ ) equals $\left(1+\kappa_{1}\right) \cdots\left(1+\kappa_{n}\right)$. In particular, this explains the statement made in [11, §1] concerning the topological dimension of the set of so-called strongly Rayleigh probability measures on the Boolean algebra $2^{[n]}$, where $[n]=\{1, \ldots, n\}$.

Lemma 3.2. Let $V \subseteq \mathbb{K}\left[z_{1}, \ldots, z_{n}\right]$ be a $\mathbb{K}$-linear space, where $\mathbb{K}=\mathbb{R}$ or $\mathbb{C}$.

(i) If $\mathbb{K}=\mathbb{R}$ and every non-zero element of $V$ is real stable then $\operatorname{dim} V \leq 2$.

(ii) If $\mathbb{K}=\mathbb{C}$ and every non-zero element of $V$ is stable then $\operatorname{dim} V \leq 1$.

Proof. We first deal with the real case. Suppose that there are three linearly independent polynomials $f_{1}, f_{2}$ and $f_{3}$ in $V$. By the multivariate Hermite-KakeyaObreschkoff theorem (Theorem 1.9) and the assumption on $V$ these polynomials are mutually in proper position. Without loss of generality we may assume that $f_{1} \ll f_{2}$ and $f_{1} \gg f_{3}$. Now consider the line segment in $V$ given by $\ell_{\theta}=\theta f_{3}+(1-\theta) f_{2}$, $0 \leq \theta \leq 1$, and note in particular that for any such $\theta$ one has either $f_{1} \ll \ell_{\theta}$ or $f_{1} \gg \ell_{\theta}$. Set $\eta=\sup \left\{\theta \in[0,1]: f_{1} \ll \ell_{\theta}\right\}$. Since $f_{1} \ll \ell_{0}$ and $f_{1} \gg \ell_{1}$ it follows from Hurwitz' theorem (Theorem 1.6) that $f_{1} \ll \ell_{\eta}$ and $f_{1} \gg \ell_{\eta}$. This means that $f_{1}$ and $\ell_{\eta}$ are constant multiples of each other (cf. Corollary 1.10), contrary to the assumption that $f_{1}, f_{2}$ and $f_{3}$ are linearly independent.

For the complex case let

$$
V_{R}=\left\{p: p+i q \in V \text { with } p, q \in \mathbb{R}\left[z_{1}, \ldots, z_{n}\right]\right\}
$$

be the "real component" of $V$. By Theorem 1.9 all non-zero polynomials in $V_{R}$ are real stable hence $\operatorname{dim}_{\mathbb{R}} V_{R} \leq 2$ by part (i) proved above. Note that $V$ is the complex span of $V_{R}$. If $\operatorname{dim}_{\mathbb{R}} V_{R} \leq 1$ we are done so we may assume that $\{p, q\}$ is 
a basis of $V_{R}$ with $f:=p+i q \in V$ which by assumption is a (not identically zero) stable polynomial. By Theorem [1.9 again we have $W_{j}[p, q](x) \geq 0,1 \leq j \leq n$, $x \in \mathbb{R}^{n}$, and since $p$ and $q$ are linearly independent the $j_{0}$-th Wronskian $W_{j_{0}}[p, q]$ is not identically zero for some index $j_{0}$. Now if $g \in V \backslash\{0, f\}$ we may write

$$
g=a p+b q+i(c p+d q)
$$

for some $a, b, c, d \in \mathbb{R}$. We have to show that $g$ is a (complex) constant multiple of $f$. Since $g \in V$ is not identically zero it is a stable polynomial, so that in particular

$$
W_{j_{0}}[a p+b q, c p+d q]=(a d-b c) W_{j_{0}}[p, q] \geq 0
$$

(cf. Theorem 1.91). Since $W_{j_{0}}[p, q] \geq 0$ and $W_{j_{0}}[p, q] \not \equiv 0$ it follows that $a d-b c \geq 0$.

Let $u, v \in \mathbb{R}$ and note that by linearity we have

$$
g+(u+i v) f=(a+u) p+(b-v) q+i((c+v) p+(d+u) q) \in V .
$$

Arguing as above we deduce that

$$
H(u, v):=(a+u)(d+u)-(b-v)(c+v) \geq 0
$$

for all $u, v \in \mathbb{R}$. But

$$
4 H(u, v)=(2 u+a+d)^{2}+(2 v+c-b)^{2}-(a-d)^{2}-(b+c)^{2},
$$

so $H(u, v) \geq 0$ for all $u, v \in \mathbb{R}$ if and only if $a=d$ and $b=-c$. This gives

$$
g=a p-c q+i(c p+a q)=(a+i c)(p+i q)=(a+i c) f,
$$

as was to be shown.

We can now show that the symbol of any stability preserver whose range is not one-dimensional must necessarily be stable.

Proof of Necessity in Theorem 1.1. Suppose that $T: \mathbb{C}_{\kappa}\left[z_{1}, \ldots, z_{n}\right] \rightarrow \mathbb{C}\left[z_{1}, \ldots, z_{n}\right]$ preserves stability. Given $W \in\{\zeta \in \mathbb{C}: \operatorname{Im}(\zeta)>0\}^{n}$ we have that $T\left[(z+W)^{\kappa}\right] \in$ $\mathcal{H}_{n}(\mathbb{C}) \cup\{0\}$. Assume first that there exists $W \in\{\zeta \in \mathbb{C}: \operatorname{Im}(\zeta)>0\}^{n}$ for which $T\left[(z+W)^{\kappa}\right] \equiv 0$ and let $f \in \mathbb{C}_{\kappa}\left[z_{1}, \ldots, z_{n}\right]$. Then by Lemma 3.1 there is an $\epsilon>0$ such that $(z+W)^{\kappa}+\epsilon f(z) \in \mathcal{H}_{n}(\mathbb{C})$. It follows that

$$
\epsilon T[f(z)]=T\left[(z+W)^{\kappa}+\epsilon f(z)\right] \in \mathcal{H}_{n}(\mathbb{C}) \cup\{0\} .
$$

Hence the image of $T$ is a linear space whose non-zero elements are all stable polynomials. By Lemma 3.2 (ii) we have that $T$ is given by $T(f)=\alpha(f) P$, where $P$ is a stable polynomial and $\alpha$ is a linear functional.

On the other hand, if $T\left[(z+W)^{\kappa}\right] \not \equiv 0$ for $W \in\{\zeta \in \mathbb{C}: \operatorname{Im}(\zeta)>0\}^{n}$ then $T\left[(z+W)^{\kappa}\right] \in \mathcal{H}_{n}(\mathbb{C})$ for all such $W$, so that $G_{T}(z, w)=T\left[(z+w)^{\kappa}\right] \in \mathcal{H}_{2 n}(\mathbb{C})$.

\section{Real Stability Preservers}

The aim of this section is to prove Theorem 1.2. Let

$$
\mathcal{H}_{n}^{-}(\mathbb{C})=\left\{f(-z): f(z) \in \mathcal{H}_{n}(\mathbb{C})\right\} .
$$

Proposition 4.1. For any $n \in \mathbb{N}$ the following holds:

$$
\mathcal{H}_{n}(\mathbb{C}) \cap \mathcal{H}_{n}^{-}(\mathbb{C})=\mathbb{C H}_{n}(\mathbb{R}):=\left\{c f: c \in \mathbb{C}, f \in \mathcal{H}_{n}(\mathbb{R})\right\} .
$$


Proof. Let $h, g \in \mathbb{R}\left[z_{1}, \ldots, z_{n}\right]$ be such that $h+i g \in \mathcal{H}_{n}(\mathbb{C}) \cap \mathcal{H}_{n}^{-}(\mathbb{C})$ and set $f=h-i g$. If $x, y \in \mathbb{R}^{n}$ then

$$
\overline{f(x+i y)}=h(-(-x+i y))+i g(-(-x+i y)) .
$$

Since $h+i g \in \mathcal{H}_{n}^{-}(\mathbb{C})$, the right-hand side of 4.1) is non-vanishing whenever $y \in \mathbb{R}_{+}^{n}$, which amounts to saying that $f(x+i y) \neq 0$ for all $y \in \mathbb{R}_{+}^{n}$. By Definition 1.1 this implies that $-g \ll h$, i.e., $h \ll g$. On the other hand, $g \ll h$ since $h+i g \in$ $\mathcal{H}_{n}(\mathbb{C})$. Therefore the proposition is just a reformulation of Corollary 1.10 .

Proof of Theorem 1.2. If $T: \mathbb{R}_{\kappa}\left[z_{1}, \ldots, z_{n}\right] \rightarrow \mathbb{R}\left[z_{1}, \ldots, z_{n}\right]$ is a linear operator as in part (a) of Theorem 1.2 then it preserves real stability (by Theorem 1.9). Moreover, if $T, T^{\prime}: \mathbb{R}_{\kappa}\left[z_{1}, \ldots, z_{n}\right] \rightarrow \mathbb{R}\left[z_{1}, \ldots, z_{n}\right]$ are linear operators whose symbols satisfy $G_{T^{\prime}}(z, w)=G_{T}(z,-w)$ then these operators are related by $T^{\prime}(f)(z)=$ $(-1)^{\kappa} T(f(-z))$, hence they preserve real stability simultaneously. To settle the sufficiency part of the theorem we may therefore assume that $G_{T}(z, w)$ is real stable. But then the desired conclusion simply follows from the complex case.

To prove the necessity part of Theorem 1.2 consider $G_{T}(z, w)=T\left[(z+w)^{\kappa}\right]$ and let $W \in\{z \in \mathbb{C}: \operatorname{Im}(z)>0\}^{n}$. Write $(z+W)^{\kappa} \in \mathcal{H}_{n}(\mathbb{C})$ as

$$
(z+W)^{\kappa}=F(z)+i G(z), \quad F, G \in \mathbb{R}_{\kappa}\left[z_{1}, \ldots, z_{n}\right] .
$$

By the multivariate Hermite-Kakeya-Obreschkoff theorem (Theorem 1.9) we have that $\alpha F+\beta G \in \mathcal{H}_{n}(\mathbb{R}) \cup\{0\}$ for all $\alpha, \beta \in \mathbb{R}$ and hence that $\alpha T(F)+\beta T(G) \in$ $\mathcal{H}_{n}(\mathbb{R}) \cup\{0\}$ for all $\alpha, \beta \in \mathbb{R}$. By Theorem 1.9 again this means that

$$
T(F)+i T(G)=G_{T}(z, W) \in \mathcal{H}_{n}(\mathbb{C}) \cup \mathcal{H}_{n}^{-}(\mathbb{C}) \cup\{0\} .
$$

Suppose that there are $W_{1}, W_{2} \in\{z \in \mathbb{C}: \operatorname{Im}(z)>0\}^{n}$ such that $G_{T}\left(z, W_{1}\right) \in$ $\mathcal{H}_{n}(\mathbb{C}) \cup\{0\}$ and $G_{T}\left(z, W_{2}\right) \in \mathcal{H}_{n}^{-}(\mathbb{C}) \cup\{0\}$. By a homotopy argument we then deduce that there exists $t \in[0,1]$ such that

$$
G_{T}\left(z, W^{\prime}\right)=\mathcal{H}_{n}(\mathbb{C}) \cap \mathcal{H}_{n}^{-}(\mathbb{C}) \cup\{0\},
$$

where $W^{\prime}=(1-t) W_{1}+t W_{2} \in\{z \in \mathbb{C}: \operatorname{Im}(z)>0\}^{n}$. Therefore, by Proposition 4.1 there is a real stable polynomial $p$ and $a, b \in \mathbb{R}$ such that $G_{T}\left(z, W^{\prime}\right)=(a+b i) p(z)$. Let us write $\left(z+W^{\prime}\right)^{\kappa}=f(z)+i g(z)$, where $f, g$ have real coefficients. Then $T(f)=a p$ and $T(g)=b p$ so that

$$
T(b f-a g)=0 .
$$

Now, as noted in the proof of Lemma 3.1 for any $h \in \mathbb{R}_{\kappa}\left[z_{1}, \ldots, z_{n}\right]$ there is an $\epsilon>0$ such that

$$
\frac{|h|}{\left|\left(z+W^{\prime}\right)^{\kappa}\right|}<\frac{1}{\epsilon}
$$

whenever $\operatorname{Im}\left(z_{i}\right) \geq 0,1 \leq i \leq n$. Hence, if $a^{2}+b^{2} \neq 0$ (the case $a=b=0$ follows similarly) we get

$$
\frac{|h|}{|b f-a g+i(a f+b g)|}=\frac{|h|}{\left|(b+i a)\left(z+W^{\prime}\right)^{\kappa}\right|}<(\epsilon|(b+i a)|)^{-1}=\frac{1}{\epsilon^{\prime}} .
$$

This implies that

$$
\left|b f-a g+\epsilon^{\prime} h+i(a f+b g)\right|>0
$$


whenever $\operatorname{Im}\left(z_{i}\right) \geq 0,1 \leq i \leq n$. In particular, $b f-a g+\epsilon^{\prime} h+i(a f+b g) \in \mathcal{H}_{n}(\mathbb{C})$ so by Theorem 1.9 we have $b f-a g+\epsilon^{\prime} h \in \mathcal{H}_{n}(\mathbb{R}) \cup\{0\}$. It follows that

$$
T(h)=\frac{1}{\epsilon^{\prime}} T\left(b f-a g+\epsilon^{\prime} h\right) \in \mathcal{H}_{n}(\mathbb{R}) \cup\{0\} .
$$

This means that all nonzero polynomials in the image of $T$ are real stable and by Lemma 3.2 we conclude that the image of $T$ is of dimension at most two.

Thus we may assume that $T\left[(z+W)^{\kappa}\right] \in \mathcal{H}_{n}(\mathbb{C})$ for all $W \in\{z \in \mathbb{C}: \operatorname{Im}(z)>0\}^{n}$ or $T\left[(z-W)^{\kappa}\right] \in \mathcal{H}_{n}(\mathbb{C})$ for all $W \in\{z \in \mathbb{C}: \operatorname{Im}(z)>0\}^{n}$. But this amounts to saying that $T\left[(z+w)^{\kappa}\right] \in \mathcal{H}_{2 n}(\mathbb{R})$ or $T\left[(z-w)^{\kappa}\right] \in \mathcal{H}_{2 n}(\mathbb{R})$, as claimed.

\section{Soft-Core/Transcendental Classifications}

In this section we settle Theorems 1.3 and 1.4 that is, the transcendental characterizations of stability, respectively real stability preservers. Let $\mathbb{K}=\mathbb{C}$ or $\mathbb{R}$. A linear operator $T: \mathbb{K}\left[z_{1}, \ldots, z_{n}\right] \rightarrow \mathbb{K}\left[z_{1}, \ldots, z_{n}\right]$ preserves stability if and only if the same holds for all its restrictions $T_{\kappa}: \mathbb{K}_{\kappa}\left[z_{1}, \ldots, z_{n}\right] \rightarrow \mathbb{K}\left[z_{1}, \ldots, z_{n}\right]$, $\kappa \in \mathbb{N}^{n}$. Therefore, by Theorem 1.1, Theorem 1.2 and the definitions of the symbols $G_{T_{\kappa}}(z, w), \bar{G}_{T}(z, w)$ we see that Theorem 1.3, respectively Theorem 1.4, would follow from the case $\mathbb{K}=\mathbb{C}$, respectively $\mathbb{K}=\mathbb{R}$, of the next theorem. As before we use the standard (product) partial order $\leq$ on $\mathbb{N}^{n}$ : for $\alpha, \beta \in \mathbb{N}^{n}$ one has $\alpha \leq \beta$ if $\alpha_{i} \leq \beta_{i}$ for all $1 \leq i \leq n$. Set $\alpha !=\alpha_{1} ! \cdots \alpha_{n}$ ! as in $\$ 1$ and let

$$
(\beta)_{\alpha}=\left\{\begin{array}{l}
\frac{\beta !}{(\beta-\alpha) !} \text { if } \alpha \leq \beta, \\
0 \text { otherwise. }
\end{array}\right.
$$

Theorem 5.1. Let $F(z, w)=\sum_{\alpha \in \mathbb{N}^{n}} P_{\alpha}(z) w^{\alpha}$, where $z=\left(z_{1}, \ldots, z_{n}\right)$ and $w=$ $\left(w_{1}, \ldots, w_{n}\right)$, be a formal power series in $w$ with coefficients in $\mathbb{K}\left[z_{1}, \ldots, z_{n}\right]$. Then $F(z, w) \in \overline{\mathcal{H}}_{2 n}(\mathbb{K})$ if and only if

$$
\sum_{\alpha \leq \beta}(\beta)_{\alpha} P_{\alpha}(z) w^{\alpha} \in \mathcal{H}_{2 n}(\mathbb{K}) \cup\{0\}
$$

for all $\beta \in \mathbb{N}^{n}$.

The proof of Theorem 5.1 requires several new ingredients and additional results that we proceed to describe. Since the arguments are the same for $\mathbb{K}=\mathbb{R}$ and $\mathbb{K}=\mathbb{C}$, we will only focus on the latter case.

5.1. Generalized Jensen Multipliers. For $\alpha, \beta \in \mathbb{N}^{n}$ let $J(\alpha, \beta)=(\beta)_{\alpha} \beta^{-\alpha}$ (we use the convention that $k^{ \pm k}=1$ for $k=0$ ). For $n=1$ these are the so-called Jensen multipliers that are known to preserve (univariate) stability [14, 32, 43]. For each fixed $\beta \in \mathbb{N}^{n}$ the sequences $\{J(\alpha, \beta)\}_{\alpha \leq \beta}$ and $\left\{(\beta)_{\alpha}\right\}_{\alpha \leq \beta}$ may be called multivariate (or generalized) Jensen multipliers in view of the following lemma.

Lemma 5.2. Let $\beta \in \mathbb{N}^{n}$. The linear operators on $\mathbb{C}\left[z_{1}, \ldots, z_{n}\right]$ defined by

$$
\begin{aligned}
& z^{\alpha} \mapsto J(\alpha, \beta) z^{\alpha}, \quad \alpha \in \mathbb{N}^{n}, \\
& z^{\alpha} \mapsto(\beta)_{\alpha} z^{\alpha}, \quad \alpha \in \mathbb{N}^{n},
\end{aligned}
$$

preserve stability. 
Proof. Fix $\beta \in \mathbb{N}^{n}$. Since the first operator is a composition of the second operator and a rescaling of the variables, it is enough to prove the lemma only for the second operator - call it $T$ and denote by $T_{\kappa}$ its restriction to $\mathbb{C}_{\kappa}\left[z_{1}, \ldots, z_{n}\right]$, where $\kappa \in \mathbb{N}^{n}$. By Theorem 1.1 we need to show that

$$
G_{T_{\kappa}}(z, w)=T_{\kappa}\left[(z+w)^{\kappa}\right]=\sum_{\alpha \leq \kappa}\left(\begin{array}{l}
\kappa \\
\alpha
\end{array}\right)(\beta)_{\alpha} z^{\alpha} w^{\kappa-\alpha}
$$

is stable for all $\kappa \in \mathbb{N}^{n}$. However,

$$
G_{T_{\kappa}}(z, w)=\prod_{i=1}^{n}\left[\sum_{j=0}^{\kappa_{i}} j !\left(\begin{array}{c}
\kappa_{i} \\
j
\end{array}\right)\left(\begin{array}{c}
\beta_{i} \\
j
\end{array}\right) z_{i}^{j} w_{i}^{\kappa_{i}-j}\right],
$$

so the lemma amounts to showing that for any $m, n \in \mathbb{N}$ the univariate polynomial

$$
g(t)=\sum_{j=0}^{n} j !\left(\begin{array}{c}
n \\
j
\end{array}\right)\left(\begin{array}{c}
m \\
j
\end{array}\right) t^{j}
$$

is real-rooted (then necessarily with all negative roots). To prove this note that

$$
t^{-m} g(t)=\left.S\left[t^{m}\right]\right|_{t \rightarrow t^{-1}}
$$

where $S$ is the operator on $\mathbb{C}[t]$ given by $S=\left(1+\frac{d}{d t}\right)^{n}$. To prove the well known fact that $S$ preserves stability it suffices to consider the case $n=1$. The symbol of $S$ is $S\left[(t+w)^{m}\right]=(m+t+w)(t+w)^{m-1}$, which is a stable polynomial. The desired conclusion now follows from Theorem 1.2 .

5.2. Multivariate Szász Principles. A remarkable fact about univariate stable polynomials, first noted by Szász [55] and subsequently used by Edrei [18], is that the growth of such a polynomial is controlled by its first few non-vanishing coefficients. We extend Szász' principle to higher dimensions and establish estimates for the growth of multivariate stable polynomials.

Lemma 5.3 (Szász). Suppose that $f(z)=1+\sum_{i=1}^{k} a_{i} z^{i}=\prod_{j=1}^{k}\left(1+\xi_{j} z\right)$ is stable. Then

$$
\sum_{j=1}^{k}\left|\xi_{j}\right|^{2} \leq 3\left|a_{1}\right|^{2}+2\left|a_{2}\right|
$$

Proof. By assumption one has $\operatorname{Im}\left(\xi_{j}\right) \leq 0,1 \leq j \leq k$, hence

$$
\sum_{j=1}^{k} \operatorname{Im}\left(\xi_{j}\right)^{2} \leq\left(\sum_{j=1}^{k} \operatorname{Im}\left(\xi_{j}\right)\right)^{2}=\operatorname{Im}\left(a_{1}\right)^{2} .
$$


Since $\sum_{j=1}^{k} \xi_{j}^{2}=a_{1}^{2}-2 a_{2}$ it follows that

$$
\begin{aligned}
\sum_{j=1}^{k}\left|\xi_{j}\right|^{2} & =\sum_{j=1}^{k}\left(\operatorname{Re}\left(\xi_{j}\right)^{2}-\operatorname{Im}\left(\xi_{j}\right)^{2}\right)+2 \sum_{j=1}^{k} \operatorname{Im}\left(\xi_{j}\right)^{2} \\
& =\operatorname{Re}\left(\sum_{j=1}^{k} \xi_{j}^{2}\right)+2 \sum_{j=1}^{k} \operatorname{Im}\left(\xi_{j}\right)^{2} \\
& =\operatorname{Re}\left(a_{1}^{2}-2 a_{2}\right)+2 \sum_{j=1}^{k} \operatorname{Im}\left(\xi_{j}\right)^{2} \\
& \leq \operatorname{Re}\left(a_{1}^{2}-2 a_{2}\right)+2 \operatorname{Im}\left(a_{1}\right)^{2} \leq 3\left|a_{1}\right|^{2}+2\left|a_{2}\right|,
\end{aligned}
$$

as claimed.

Szász used his lemma to obtain the following bound for the polynomial $f$ in Lemma 5.3 .

$$
|f(z)| \leq \exp \left(r\left|a_{1}\right|+3 r^{2}\left|a_{1}\right|^{2}+3 r^{2}\left|a_{2}\right|\right), \quad|z| \leq r .
$$

Remark 5.1. If the univariate polynomial $f$ in Lemma 5.3 is assumed to have all real and negative zeros then $\sum_{j=1}^{k} \xi_{j}^{2} \leq a_{1}^{2}$. Using the latter inequality in the following arguments one can get stronger bounds for the growth of multivariate real stable polynomials with all non-negative coefficients.

As before, we let $\left\{e_{i}\right\}_{i=1}^{n}$ be the standard basis in $\mathbb{R}^{n}$.

Lemma 5.4. Suppose that the polynomial

$$
f(z)=1+\sum_{|\beta|>0} a(\beta) z^{\beta} \in \mathbb{C}\left[z_{1}, \ldots, z_{n}\right]
$$

is stable and let

$$
A=\left[3\left(\sum_{i=1}^{n}\left|a\left(e_{i}\right)\right|\right)^{2}+2 \sum_{i, j=1}^{n} \mid a\left(e_{i}+e_{j}\right)\right]^{1 / 2} .
$$

Then

$$
|a(\beta)| \leq|\beta|^{-|\beta| / 2} \frac{\beta^{\beta}}{\beta !} A^{|\beta|}, \quad \beta=\left(\beta_{1}, \ldots, \beta_{n}\right) \in \mathbb{N}^{n},
$$

where $|\beta|=\sum_{i=1}^{n} \beta_{i}$.

Proof. Let us rewrite the above polynomial as $f(z)=\sum_{\alpha} a(\alpha) z^{\alpha}$ and suppose that $\beta \neq 0$ is such that $a(\beta) \neq 0$. Since the operator $z^{\alpha} \mapsto J(\alpha, \beta) z^{\alpha}, \alpha \in \mathbb{N}^{n}$, preserves stability (Lemma 5.2) the polynomial

$$
J(\beta, \beta) a(\beta) z^{\beta}+\sum_{0<|\alpha|<|\beta|} J(\alpha, \beta) a(\alpha) z^{\alpha}+1
$$

is stable. Setting all variables equal to $t$ we deduce that the univariate polynomial $g(t)$ of degree $k=|\beta|$ given by

$$
g(t)=\frac{\beta !}{\beta^{\beta}} a(\beta) t^{k}+\sum_{2<|\alpha|<k} J(\alpha, \beta) a(\alpha) t^{|\alpha|}
$$




$$
+\left[\sum_{i \neq j: \beta_{i} \beta_{j} \geq 1} a\left(e_{i}+e_{j}\right)+\sum_{i: \beta_{i} \geq 2}\left(1-1 / \beta_{i}\right) a\left(2 e_{i}\right)\right] t^{2}+\sum_{i: \beta_{i} \geq 1} a\left(e_{i}\right) t+1
$$

is stable. If we rewrite $g(t)=\sum_{i=0}^{k} a_{i} t^{i}=\prod_{j=1}^{k}\left(1+\xi_{j} t\right)$ then by Lemma 5.3 we get

$$
\left|\frac{\beta !}{\beta^{\beta}} a(\beta)\right|=\prod_{j=1}^{k}\left|\xi_{j}\right| \leq\left[\frac{\sum_{j=1}^{k}\left|\xi_{j}\right|^{2}}{k}\right]^{k / 2} \leq\left(3\left|a_{1}\right|^{2}+2\left|a_{2}\right|\right)^{k / 2} k^{-k / 2} \leq A^{k} k^{-k / 2},
$$

which is the desired estimate.

Theorem 5.5. Suppose that the polynomial

$$
f(z)=1+\sum_{|\beta|>0} a(\beta) z^{\beta} \in \mathbb{C}\left[z_{1}, \ldots, z_{n}\right]
$$

is stable and let

$$
\begin{aligned}
& B=2^{n-1} \frac{\sqrt{2 e^{2}-e}}{e-1}=2^{n-1} \cdot 2.0210 \ldots \\
& C=6 e^{2}\left(\sum_{i=1}^{n}\left|a\left(e_{i}\right)\right|\right)^{2}+4 e^{2} \sum_{i, j=1}^{n}\left|a\left(e_{i}+e_{j}\right)\right| .
\end{aligned}
$$

Then

$$
\max \left\{|f(z)|:\left|z_{i}\right| \leq r, 1 \leq i \leq n\right\} \leq B e^{C r^{2}}, \quad r \geq 0 .
$$

Proof. We make use of the following simple inequalities that may be proved by induction or from Stirling estimates.

$$
e^{-n} \leq \frac{n !}{n^{n}} \leq(e n+1) e^{-n}, \quad n \geq 0 .
$$

Let $d(n, k)=\sum_{\beta \in \mathbb{N}^{n},|\beta|=k} \beta^{\beta} / \beta$ ! and note that $\left|\left\{\beta \in \mathbb{N}^{n}:|\beta|=k\right\}\right|=\left(\begin{array}{c}n+k-1 \\ k\end{array}\right)$ for $n, k \in \mathbb{N}$. By (5.1) we have the following (rough) estimate:

$$
d(n, k) \leq\left(\begin{array}{c}
n+k-1 \\
k
\end{array}\right) e^{k} \leq 2^{n-1+k} e^{k}, \quad n, k \in \mathbb{N} .
$$

Let $A$ be as in Lemma 5.4 and apply the same lemma:

$$
\begin{aligned}
& \max \left\{|f(z)|:\left|z_{i}\right| \leq r, 1 \leq i \leq n\right\} \leq \sum_{\beta \in \mathbb{N}^{n}}|\beta|^{-|\beta| / 2} \frac{\beta^{\beta}}{\beta !}(A r)^{|\beta|} \\
& =\sum_{k=0}^{\infty} d(n, k) k^{-k / 2}(A r)^{k} \leq 2^{n-1} \sum_{k=0}^{\infty} k^{-k / 2}(2 e A r)^{k} .
\end{aligned}
$$

Set $R=2 e A r$. By (5.1) and the Cauchy-Schwarz inequality we get

$$
\begin{aligned}
\sum_{k=0}^{\infty} k^{-k / 2} R^{k} \leq \sum_{k=0}^{\infty} & \sqrt{(e k+1) e^{-k}} \cdot \sqrt{\frac{R^{2 k}}{k !}} \\
& \leq \sqrt{\left(\sum_{k=0}^{\infty}(e k+1) e^{-k}\right) \cdot\left(\sum_{k=0}^{\infty} \frac{R^{2 k}}{k !}\right)}=\frac{\sqrt{2 e^{2}-e}}{e-1} \cdot e^{R^{2} / 2},
\end{aligned}
$$

which combined with (5.2) proves the theorem. 
Given $f(z)=\sum_{\alpha} a(\alpha) z^{\alpha} \in \mathbb{C}\left[z_{1}, \ldots, z_{n}\right]$ let $\operatorname{supp}(f)=\left\{\alpha \in \mathbb{N}^{n}: a(\alpha) \neq 0\right\}$ be its support. Denote by $\mathcal{M}(f)$ the set of minimal elements of $\operatorname{supp}(f)$ with respect to the partial order $\leq$ on $\mathbb{N}^{n}$. Moreover, for a fixed set $\mathcal{M} \subset \mathbb{N}^{n}$ we let

$$
\mathcal{M}_{\ell}=\left\{\alpha+\beta: \alpha \in \mathcal{M}, \beta \in \mathbb{N}^{n},|\beta| \leq \ell\right\} .
$$

We can now establish a multivariate Szász principle.

Theorem 5.6. Let $\mathcal{M} \subset \mathbb{N}^{n}$ be a finite non-empty set and $f(z)=\sum_{\alpha} a(\alpha) z^{\alpha} \in$ $\mathbb{C}\left[z_{1}, \ldots, z_{n}\right]$ be a stable polynomial with $\mathcal{M}(f)=\mathcal{M}$. Then there are constants $B$ and $C$ depending only on the coefficients $a(\alpha)$ with $\alpha \in \mathcal{M}_{2}$ such that

$$
\max \left\{|f(z)|:\left|z_{i}\right| \leq r, 1 \leq i \leq n\right\} \leq B e^{C r^{2}}
$$

for all $r \geq 0$. Moreover, $B$ and $C$ can be chosen so that they depend continuously on the aforementioned set of coefficients.

Proof. We construct $B$ and $C$ inductively over $m=n+k$, where

$$
k=\max \{|\alpha|: \alpha \in \mathcal{M}(f)\} .
$$

If $f(0) \neq 0$ then $k=0$ and the bound follows from Theorem 5.5. so we may assume that $k>0$. Also, if $n=1$ we may just factor out a multiple of $z$ and use the fact that $r \leq e^{r^{2}}$ together with Theorem 5.5. Hence we may assume that $n>1$.

Let $\alpha=\left(\alpha_{1}, \ldots, \alpha_{n}\right) \in \mathcal{M}(f)$ be such that $|\alpha|=k$ and suppose (without loss of generality) that $\alpha_{1}>0$. Now

$$
f\left(z_{1}, \ldots, z_{n}\right)=f\left(0, z_{2}, \ldots, z_{n}\right)+z_{1} \int_{0}^{1} \frac{\partial f}{\partial z_{1}}\left(z_{1} t, z_{2}, \ldots, z_{n}\right) d t
$$

so that

$$
\left|f\left(z_{1}, \ldots, z_{n}\right)\right|_{r} \leq\left|f\left(0, z_{2}, \ldots, z_{n}\right)\right|_{r}+r\left|\frac{\partial f}{\partial z_{1}}\right|_{r},
$$

where $|g|_{r}=\max \left\{|g(z)|:\left|z_{i}\right| \leq r, 1 \leq i \leq n\right\}$ for $g \in \mathbb{C}\left[z_{1}, \ldots, z_{n}\right]$. Since

$$
\max \left\{|\beta|: \beta \in \mathcal{M}\left(\partial f / \partial z_{1}\right)\right\}=k-1
$$

and $f\left(0, z_{2}, \ldots, z_{n}\right)$ is either identically zero or a stable polynomial in $n-1$ variables, by the induction assumption we have

$$
\left|f\left(0, z_{2}, \ldots, z_{n}\right)\right|_{r} \leq B_{1} e^{C_{1} r^{2}} \quad \text { and } \quad\left|\frac{\partial f}{\partial z_{1}}\right|_{r} \leq B_{2} e^{C_{2} r^{2}} .
$$

Set $C=\max \left\{C_{1}, C_{2}+1\right\}$ and $B=2 \max \left\{B_{1}, B_{2}\right\}$. From the above estimates we get $|f|_{r} \leq B e^{C r^{2}}$, which completes the induction step.

5.3. Proof of Theorem [5.1. We can now settle Theorem 5.1 and thereby - as explained at the beginning of this section - Theorems 1.3 and 1.4 as well. Note that the case $\mathbb{K}=\mathbb{R}$ in Theorem 5.1 follows from the case $\mathbb{K}=\mathbb{C}$ since $\mathcal{H}_{2 n}(\mathbb{C}) \cap$ $\mathbb{R}\left[z_{1}, \ldots, z_{n}, w_{1}, \ldots, w_{n}\right]=\mathcal{H}_{n}(\mathbb{R})$. Therefore we assume that $\mathbb{K}=\mathbb{C}$.

Suppose that $F(z, w)=\sum_{\alpha \in \mathbb{N}^{n}} P_{\alpha}(z) w^{\alpha} \in \overline{\mathcal{H}}_{2 n}(\mathbb{C})$ and $P_{\alpha} \in \mathbb{C}\left[z_{1}, \ldots, z_{n}\right]$, $\alpha \in \mathbb{N}^{n}$. If $F_{m}(z, w)=\sum_{\alpha \in \mathbb{N}^{n}} P_{m, \alpha}(z) w^{\alpha}$ is a sequence of polynomials in $\mathcal{H}_{2 n}(\mathbb{C})$ converging to $F(z, w)$, uniformly on compacts, then $P_{m, \alpha}(z) \rightarrow P_{\alpha}(z)$ as $m \rightarrow$ $\infty$ uniformly on compacts for fixed $\alpha \in \mathbb{N}^{n}$. Given $\beta \in \mathbb{N}^{n}$ let $\Lambda$ be the linear operator on $\mathbb{C}\left[w_{1}, \ldots, w_{n}\right]$ defined by $w^{\alpha} \mapsto(\beta)_{\alpha} w^{\alpha}, \alpha \in \mathbb{N}^{n}$, and extend it to a linear operator $\bar{\Lambda}$ on $\mathbb{C}\left[z_{1}, \ldots, z_{n}, w_{1}, \ldots, w_{n}\right]$ by letting $\bar{\Lambda}\left[z^{\gamma} w^{\alpha}\right]=z^{\gamma} \Lambda\left[w^{\alpha}\right]$, $\alpha, \gamma \in \mathbb{N}^{n}$. We claim that $\bar{\Lambda}$ preserves stability in $2 n$ variables. Indeed, this amounts 
to saying that its restriction $\bar{\Lambda}_{\kappa}$ to $\mathbb{C}_{\kappa}\left[z_{1}, \ldots, z_{n}, w_{1}, \ldots, w_{n}\right]$ preserves stability for each $\kappa \in \mathbb{N}^{2 n}$. The latter statement is an immediate consequence of the fact that $\Lambda$ preserves stability in $n$ variables (by Lemma [5.2) combined with Theorem 1.1 and a straightforward computation showing that the symbol $G_{\bar{\Lambda}_{\kappa}}$ of $\bar{\Lambda}_{\kappa}$ is a stable polynomial in $4 n$ variables. Now, since $\bar{\Lambda}$ sends all but finitely many monomials $w^{\alpha}$ to 0 we have that $\bar{\Lambda}\left[F_{m}(z, w)\right] \rightarrow \bar{\Lambda}[F(z, w)]$ as $m \rightarrow \infty$, uniformly on compacts, which gives $\bar{\Lambda}[F(z, w)] \in \mathcal{H}_{2 n}(\mathbb{C}) \cup\{0\}$. This proves the necessity part of Theorem 5.1 .

To prove the converse assume that $\sum_{\alpha \leq \beta}(\beta)_{\alpha} P_{\alpha}(z) w^{\alpha} \in \mathcal{H}_{2 n}(\mathbb{C}) \cup\{0\}$ for all $\beta \in \mathbb{N}^{n}$. Let $m \in \mathbb{N}$, set $\beta_{m}=(m, \ldots, m)$ and note that

$$
F_{m}(z, w):=\sum_{\alpha \leq \beta_{m}} J\left(\alpha, \beta_{m}\right) P_{\alpha}(z) w^{\alpha}=\sum_{\alpha \leq \beta_{m}}\left(\beta_{m}\right)_{\alpha} P_{\alpha}(z)\left(\frac{w}{m}\right)^{\alpha} \in \mathcal{H}_{2 n}(\mathbb{C}) \cup\{0\} .
$$

We want to show that there is a positive integer $M$ such that for all $r>0$ there is a constant $K_{r}$ (depending only on $r$ ) such that for all $m>M$ we have $\left|F_{m}(z, w)\right| \leq K_{r}$ whenever $\left|w_{j}\right| \leq r$ and $\left|z_{j}\right| \leq r$ for $1 \leq j \leq n$. This will prove the sufficiency part of Theorem 5.1 since $\left\{F_{m}(z, w)\right\}_{m \in \mathbb{N}}$ is then a normal family whose convergent subsequences converge to $F(z, w)$ (by the fact that $\lim _{m \rightarrow \infty} J\left(\alpha, \beta_{m}\right)=1$ for all $\left.\alpha \in \mathbb{N}^{n}\right)$. Now, there exists a positive integer $N$ such that the set $\mathcal{M}\left(F_{m}\right)$ of minimal elements of $\operatorname{supp}\left(F_{m}\right)$ does not change for $m \geq N$ (this is easily seen from the fact that all lower degree terms are multiplied by eventually non-zero numbers $\left.J\left(\alpha, \beta_{m}\right)\right)$. Since $\lim _{m \rightarrow \infty} J\left(\alpha, \beta_{m}\right)=1$ it follows from Theorem 5.6 that there is a positive integer $M$ and constants $B$ and $C$ such that if $\left|w_{j}\right| \leq r$ and $\left|z_{j}\right| \leq r$, $1 \leq j \leq n$, then

$$
\left|F_{m}(z, w)\right| \leq B e^{C r^{2}}, \quad m>M
$$

which completes the proof of the theorem.

\section{Circular Domains and Lee-Yang Polynomials}

We will now solve Problems 1 2 whenever $\Omega$ is a product of arbitrary open circular domains. Using this we then characterize all linear transformations preserving the class of Lee-Yang polynomials defined with respect to any such set $\Omega$.

6.1. Products of Open Circular Domains. Recall that a Möbius transformation is a bijective conformal map of the extended complex plane $\widehat{\mathbb{C}}$ given by

$$
\phi(\zeta)=\frac{a \zeta+b}{c \zeta+d}, \quad a, b, c, d \in \mathbb{C}, a d-b c=1 .
$$

Note that one usually has the weaker requirement $a d-b c \neq 0$ but this is equivalent to (6.1) which proves to be more convenient. An open circular domain is the image (in $\mathbb{C}$ ) of the upper half-plane $H$ under a Möbius transformation, i.e., an open disk, the complement of a closed disk or an open affine half-plane.

As usual, the support of a polynomial $f(z)=\sum_{\alpha \in \mathbb{N}^{n}} a(\alpha) z^{\alpha} \in \mathbb{C}\left[z_{1}, \ldots, z_{n}\right]$, i.e., the set $\left\{\alpha \in \mathbb{N}^{n}: a(\alpha) \neq 0\right\}$, is denoted by $\operatorname{supp}(f)$. We say that $f$ is of degree $\kappa=\left(\kappa_{1}, \ldots, \kappa_{n}\right) \in \mathbb{N}^{n}$ if $\operatorname{deg}_{z_{i}}(f)=\kappa_{i}, i \in[n]$, where as before $[n]=\{1, \ldots, n\}$.

To deal with discs and exterior of discs we need some auxiliary results.

Lemma 6.1. Let $\left\{C_{i}\right\}_{i=1}^{n}$ be a family of circular domains, $f \in \mathbb{C}\left[z_{1}, \ldots, z_{n}\right]$ be of degree $\kappa \in \mathbb{N}^{n}$, and $J \subseteq[n]$ a (possibly empty) set such that $C_{j}$ is the exterior of a disk whenever $j \in J$. Denote by $g$ the polynomial in the variables $z_{j}, j \in J$, 
obtained by setting $z_{i}=c_{i} \in C_{i}$ arbitrarily for $i \notin J$. If $f$ is $C_{1} \times \cdots \times C_{n}$-stable then $\operatorname{supp}(g)$ has a unique maximal element $\gamma$ with respect to the standard partial order on $\mathbb{N}^{J}$. Moreover, $\gamma$ is the same for all choices of $c_{i} \in C_{i}, i \notin J$.

Proof. Let us first prove the lemma in the case when $J=[n]$. We may assume that $C_{j}$ is the exterior of the closed unit disk $\overline{\mathbb{D}}$ for all $j \in[n]$. Suppose that there is no unique maximal element of $\operatorname{supp}(f)$. For fixed $\lambda=\left(\lambda_{1}, \ldots, \lambda_{n}\right) \in \mathbb{R}_{+}^{n}$ and $\beta=\left(\beta_{1}, \ldots, \beta_{n}\right) \in \mathbb{N}^{n}$ consider the univariate polynomial

$$
F(\lambda, \beta ; t):=K f\left(\lambda_{1} t^{\beta_{1}}, \ldots, \lambda_{n} t^{\beta_{n}}\right),
$$

where $K=K(\lambda, \beta) \in \mathbb{C}$ is the appropriate constant making $F$ monic. Let $\mathcal{A}(\beta)$ be the closure (with respect to uniform convergence on compacts) of the set $\{F(\lambda, \beta ; t)$ : $\left.\lambda \in(1, \infty)^{n}\right\}$. By Hurwitz' theorem (Theorem 1.6) all elements of $\mathcal{A}(\beta)$ are $\mathbb{C} \backslash \overline{\mathbb{D}}$ stable. However, since there is no unique maximal element of $\operatorname{supp}(f)$ there exists $\beta \in \mathbb{N}^{n}$ such that $\mathcal{A}(\beta)$ contains polynomials of different degrees. Hence, as we vary $\lambda$ at least one zero must escape to infinity. This is impossible since all the zeros of $F(\lambda, \beta ; t)$ have modulus at most one.

If $J \neq[n]$ and the maximal elements are different for different choices of $c_{i}$ then as above some zero of a specialization of $f$ would escape to infinity contradicting the boundedness of the zeros.

When dealing with non-convex circular domains one has to be careful with degrees. We are therefore led to consider the following classes of polynomials.

Notation 6.1. If $\left\{C_{i}\right\}_{i=1}^{n}$ is a family of circular domains and $\kappa=\left(\kappa_{1}, \ldots, \kappa_{n}\right) \in$ $\mathbb{N}^{n}$ we let $\mathcal{N}_{\kappa}\left(C_{1}, \ldots, C_{n}\right)$ denote the set of $C_{1} \times \cdots \times C_{n}$-stable polynomials in $\mathbb{C}_{\kappa}\left[z_{1}, \ldots, z_{n}\right]$ that have degree $\kappa_{j}$ in $z_{j}$ whenever $C_{j}$ is non-convex. (If all $C_{j}$ are convex then $\mathcal{N}_{\kappa}\left(C_{1}, \ldots, C_{n}\right)$ is just the set of all $C_{1} \times \cdots \times C_{n}$-stable polynomials in $\mathbb{C}_{\kappa}\left[z_{1}, \ldots, z_{n}\right]$.) Let also

$$
\mathcal{N}\left(C_{1}, \ldots, C_{n}\right)=\bigcup_{\kappa \in \mathbb{N}^{n}} \mathcal{N}_{\kappa}\left(C_{1}, \ldots, C_{n}\right) .
$$

Lemma 6.2. Suppose that $C_{1}, \ldots, C_{n}, D_{1}, \ldots, D_{n}$ are open circular domains and $\kappa=\left(\kappa_{1}, \ldots, \kappa_{n}\right) \in \mathbb{N}^{n}$. Then there are Möbius transformations

$$
\zeta \mapsto \phi_{i}(\zeta)=\frac{a_{i} \zeta+b_{i}}{c_{i} \zeta+d_{i}}, \quad i \in[n],
$$

as in (6.1) such that the (invertible) linear transformation $\Phi_{\kappa}: \mathbb{C}_{\kappa}\left[z_{1}, \ldots, z_{n}\right] \rightarrow$ $\mathbb{C}_{\kappa}\left[z_{1}, \ldots, z_{n}\right]$ defined by

$$
\Phi_{\kappa}(f)\left(z_{1}, \ldots, z_{n}\right)=\left(c_{1} z_{1}+d_{1}\right)^{\kappa_{1}} \cdots\left(c_{n} z_{n}+d_{n}\right)^{\kappa_{n}} f\left(\phi_{1}\left(z_{1}\right), \ldots, \phi_{n}\left(z_{n}\right)\right)
$$

restricts to a bijection between $\mathcal{N}_{\kappa}\left(C_{1}, \ldots, C_{n}\right)$ and $\mathcal{N}_{\kappa}\left(D_{1}, \ldots, D_{n}\right)$.

Proof. The relation between tuples of open circular domains defined by relating tuples for which there is such a linear transformation $\Phi_{\kappa}$ is an equivalence relation. Therefore it suffices to assume that $C_{j}=D_{j}$ for $j \geq 2$ and that $D_{1}=H\left(=\mathbb{H}_{0}\right)$. Thus we want to find $\Phi_{\kappa}$ of the form

$$
\Phi_{\kappa}(f)=\left(c z_{1}+d\right)^{\kappa_{1}} f\left(\frac{a z_{1}+b}{c z_{1}+d}, z_{2}, \ldots, z_{n}\right) .
$$


If $C_{1}$ is convex we may chose $\phi(z)=(a z+b) /(c z+d)$ as in (6.1) so that $\phi(H)=C_{1}$ and $c z+d \neq 0$ for all $z \in H$. If $C_{1}$ is non-convex we may chose $\phi$ so that $\phi(H \backslash\{\eta\})=C_{1}$ for some specific point $\eta \in H$, namely $\eta=-d / c$.

Now let $f \in \mathcal{N}_{\kappa}\left(C_{1}, \ldots, C_{n}\right)$ and $\xi=\left(\xi_{1}, \ldots, \xi_{n}\right) \in D_{1} \times \cdots \times D_{n}$. If $c \xi_{1}+d \neq 0$ then $\phi\left(\xi_{1}\right) \in C_{1}$ and $\Phi_{\kappa}(f)(\xi) \neq 0$ by construction. If $c \xi_{1}+d=0$ then $C_{1}$ is nonconvex so by Lemma 6.1 the polynomial $z_{1} \mapsto f\left(z_{1}, \xi_{2}, \xi_{3}, \ldots, \xi_{n}\right)$ may be written as $\sum_{k=0}^{\kappa_{1}} \alpha_{k} z_{1}^{k}$ with $\alpha_{\kappa_{1}} \neq 0$. But then

$$
\Phi_{\kappa}(f)\left(\xi_{1}, \ldots, \xi_{n}\right)=\alpha_{\kappa_{1}}(a d-b c)^{\kappa_{1}} / c^{\kappa_{1}} \neq 0 .
$$

Hence $\Phi_{\kappa}(f) \in \mathcal{N}_{\kappa}\left(D_{1}, \ldots, D_{n}\right)$.

If $g \in \mathcal{N}_{\kappa}\left(D_{1}, \ldots, D_{n}\right)$ consider

$$
\Phi_{\kappa}^{-1}(g)=\left(-c z_{1}+a\right)^{\kappa_{1}} g\left(\frac{d z_{1}-b}{-c z_{1}+a}, z_{2}, \ldots, z_{n}\right),
$$

where $\phi(z)=(a z+b) /(c z+d)$ is chosen as above. It follows that $-c z+d \neq 0$ for all $z \in C_{1}$ so it only remains to prove that the degree of $\Phi_{\kappa}^{-1}(g)$ in $z_{1}$ is $\kappa_{1}$ when $C_{1}$ is non-convex. To do this fix $\xi_{j} \in D_{j}, 2 \leq j \leq n$, and consider the $H$-stable polynomial $g\left(z_{1}, \xi_{2}, \ldots, \xi_{n}\right)=\sum_{k=0}^{\kappa_{1}} a_{k} z_{1}^{k}$. The coefficient in front of $z_{1}^{\kappa_{1}}$ in $\Phi_{\kappa}^{-1}(g)\left(z_{1}, \xi_{2}, \ldots, \xi_{n}\right)$ is $(-c)^{\kappa_{1}} \sum_{k=0}^{\kappa_{1}} a_{k}(-d / c)^{k}$ which is non-zero since as we previously noted one has $-d / c \in H$.

The following theorem answers a more precise version of Problem 1 for $\mathbb{K}=\mathbb{C}$ and $\Omega=C_{1} \times \cdots \times C_{n}$, where the $C_{i}$ are arbitrary open circular domains.

Theorem 6.3. Let $\kappa \in \mathbb{N}^{n}$ and $T: \mathbb{C}_{\kappa}\left[z_{1}, \ldots, z_{n}\right] \rightarrow \mathbb{C}\left[z_{1}, \ldots, z_{n}\right]$ be a linear operator. Let further $C_{i}, i \in[n]$, be open circular domains given by $C_{i}=\phi_{i}^{-1}(H)$, $i \in[n]$, where $\phi_{i}, i \in[n]$, are Möbius transformations as in (6.2) such that the corresponding linear transformation $\Phi_{\kappa}$ defined in (6.3) restricts to a bijection between $\mathcal{N}_{\kappa}(H, \ldots, H)$ and $\mathcal{N}_{\kappa}\left(C_{1}, \ldots, C_{n}\right)$ (cf. Lemma 6.2). Then

$$
T: \mathcal{N}_{\kappa}\left(C_{1}, \ldots, C_{n}\right) \rightarrow \mathcal{N}\left(C_{1}, \ldots, C_{n}\right) \cup\{0\}
$$

if and only if either

(a) $T$ has range of dimension at most one and is of the form

$$
T(f)=\alpha(f) P,
$$

where $\alpha$ is a linear functional on $\mathbb{C}_{\kappa}\left[z_{1}, \ldots, z_{n}\right]$ and $P$ is a $C_{1} \times \cdots \times C_{n^{-}}$ stable polynomial, or

(b) the polynomial in $2 n$ variables $z_{1}, \ldots, z_{n}, w_{1}, \ldots, w_{n}$ given by

$$
T\left[\prod_{i=1}^{n}\left(\left(a_{i} z_{i}+b_{i}\right)\left(c_{i} w_{i}+d_{i}\right)+\left(a_{i} w_{i}+b_{i}\right)\left(c_{i} z_{i}+d_{i}\right)\right)^{\kappa_{i}}\right]
$$

is $C_{1} \times \cdots \times C_{n} \times C_{1} \times \cdots \times C_{n}$-stable.

Remark 6.1. By analogy with the definitions made in $\$ 1.1$ we may call the polynomial in Theorem 6.3 (b) the algebraic symbol of $T$ with respect to $C_{1} \times \cdots \times C_{n}$. Note that in the case of the open unit disk $C_{i}=\mathbb{D}, i \in[n]$, or the exterior of the closed unit disk $C_{i}=\mathbb{C} \backslash \overline{\mathbb{D}}, i \in[n]$, this symbol becomes a constant multiple of $T\left[(1+z w)^{\kappa}\right]$ while in the case of an open half-plane bordering on the origin it is just a constant multiple of $G_{T}(z, w)=T\left[(z+w)^{\kappa}\right]$. For the open right half-plane $\mathbb{H}_{\frac{\pi}{2}}$ it is often more convenient (but equivalent) to choose the symbol $T\left[(1+z w)^{\kappa}\right]$. 
Proof of Theorem [6.3. Let $T: \mathbb{C}_{\kappa}\left[z_{1}, \ldots, z_{n}\right] \rightarrow \mathbb{C}\left[z_{1}, \ldots, z_{n}\right]$ be a linear operator and $\gamma=\left(\gamma_{1}, \ldots, \gamma_{n}\right) \in \mathbb{N}^{n}$ be such that $T\left(\mathbb{C}_{\kappa}\left[z_{1}, \ldots, z_{n}\right]\right) \subseteq \mathbb{C}_{\gamma}\left[z_{1}, \ldots, z_{n}\right]$. The theorem is trivial if the range of $T$ has dimension at most one so we may assume that this is not the case. With $\phi_{i}, i \in[n]$, and $\Phi_{\kappa}$ as in the statement of the theorem let $\Phi_{\gamma}: \mathbb{C}_{\gamma}\left[z_{1}, \ldots, z_{n}\right] \rightarrow \mathbb{C}_{\gamma}\left[z_{1}, \ldots, z_{n}\right]$ be the linear transformation defined as in (6.3) only with $\gamma=\left(\gamma_{1}, \ldots, \gamma_{n}\right)$ instead of $\kappa=\left(\kappa_{1}, \ldots, \kappa_{n}\right)$. Set $T^{\prime}:=\Phi_{\gamma}^{-1} T \Phi_{\kappa}$. By Theorem 1.1 and Lemma 6.2 we have the following chain of equivalences:

$$
\begin{aligned}
T\left(\mathcal{N}_{\kappa}\left(C_{1}, \ldots, C_{n}\right)\right) \subset \mathcal{N}\left(C_{1}, \ldots, C_{n}\right) \cup\{0\} & \Longleftrightarrow \\
T^{\prime} \text { preserves }(H \text {-) stability } & \Longleftrightarrow \\
G(z, w):=T^{\prime}\left[(z+w)^{\kappa}\right] \text { is }(H \text { - }) \text { stable } & \Longleftrightarrow \\
\Phi_{\gamma \oplus \kappa}(G) \text { is } C_{1} \times \cdots \times C_{n} \times C_{1} \times \cdots \times C_{n} \text {-stable, } &
\end{aligned}
$$

where $\Phi_{\gamma \oplus \kappa}: \mathbb{C}_{\gamma \oplus \kappa}\left[z_{1}, \ldots, z_{n}, w_{1}, \ldots, w_{n}\right] \rightarrow \mathbb{C}_{\gamma \oplus \kappa}\left[z_{1}, \ldots, z_{n}, w_{1}, \ldots, w_{n}\right]$ is given by

$$
\begin{aligned}
& \Phi_{\gamma \oplus \kappa}(f)\left(z_{1}, \ldots, z_{n}, w_{1}, \ldots, w_{n}\right) \\
& \quad=\prod_{i=1}^{n}\left(c_{i} z_{i}+d_{i}\right)^{\gamma_{i}} \prod_{i=1}^{n}\left(c_{i} w_{i}+d_{i}\right)^{\kappa_{i}} f\left(\phi_{1}\left(z_{1}\right), \ldots, \phi_{n}\left(z_{n}\right), \phi_{1}\left(w_{1}\right), \ldots, \phi_{n}\left(w_{n}\right)\right),
\end{aligned}
$$

see (6.3). Now $\Phi_{\gamma \oplus \kappa}(G)$ is precisely the polynomial in Theorem 6.3 (b). Indeed, letting subscript $z$ and subscript $w$ indicate that the corresponding operator acts on the $z$ and $w$ variables, respectively, we have

$$
\begin{aligned}
\Phi_{\gamma \oplus \kappa}(G)(z, w) & =\left(\Phi_{\kappa, w} \circ \Phi_{\gamma, z} \circ \Phi_{\gamma, z}^{-1} \circ T_{z} \circ \Phi_{\kappa, z}\right)\left[(z+w)^{\kappa}\right] \\
& =T\left[\Phi_{\kappa \oplus \kappa}\left((z+w)^{\kappa}\right)\right],
\end{aligned}
$$

which completes the proof of the theorem.

Note that if all $C_{i}$ are open convex circular domains then Theorem 6.3 answers Problem 1 (for such domains) precisely as stated in the introduction, and when non-convex circular domains are involved it answers a more precise version of the problem.

6.2. Linear Preservers of Lee-Yang Polynomials. Given an open circular domain $C \subset \mathbb{C}$ let $C^{r}$ be the interior of the complement of $C$, that is, $C^{r}=\mathbb{C} \backslash \bar{C}$.

Definition 6.1. Let $\kappa=\left(\kappa_{1}, \ldots, \kappa_{n}\right) \in \mathbb{N}^{n}$ and $\left\{C_{i}\right\}_{i=1}^{n}$ be a family of open circular domains. The set of $\kappa$-Lee-Yang polynomials with respect to $\left\{C_{i}\right\}_{i=1}^{n}$ is

$$
\mathcal{L} \mathcal{Y}_{\kappa}\left(C_{1}, \ldots, C_{n}\right)=\mathcal{N}_{\kappa}\left(C_{1}, \ldots, C_{n}\right) \cap \mathcal{N}_{\kappa}\left(C_{1}^{r}, \ldots, C_{n}^{r}\right) .
$$

A Lee-Yang polynomial with respect to $\left\{C_{i}\right\}_{i=1}^{n}$ is an element of the set

$$
\mathcal{L Y}\left(C_{1}, \ldots, C_{n}\right):=\bigcup_{\kappa \in \mathbb{N}^{n}} \mathcal{L} \mathcal{Y}_{\kappa}\left(C_{1}, \ldots, C_{n}\right) .
$$

This definition is motivated by the original Lee-Yang theorem 31] and its various versions such as the so-called "circle theorem" (6, Theorem 8.4], 23, [49]). For instance, the class of "complex Lee-Yang polynomials" related to the latter which was introduced and studied in [3] corresponds to the case when $C_{i}=\mathbb{D}, i \in[n]$, in Definition 6.1. Note also that by Corollary 1.10 the Lee-Yang polynomials with 
respect to $\left\{H_{i}\right\}_{i=1}^{n}$, where $H_{i}=H, i \in[n]$, are precisely the polynomials that are non-zero complex constant multiples of real stable polynomials.

We say that a linear operator $T: \mathbb{C}_{\kappa}\left[z_{1}, \ldots, z_{n}\right] \rightarrow \mathbb{C}\left[z_{1}, \ldots, z_{n}\right]$ preserves the $\kappa$ Lee-Yang property with respect to $\left\{C_{i}\right\}_{i=1}^{n}$ if $T(f) \in \mathcal{L} \mathcal{Y}\left(C_{1}, \ldots, C_{n}\right) \cup\{0\}$ whenever $f \in \mathcal{L Y}_{\kappa}\left(C_{1}, \ldots, C_{n}\right)$. From our symbol theorems we can deduce a characterization of all such linear operators. We only display the case when $T$ is non-degenerate in the sense that its image has dimension larger than 2 .

Theorem 6.4. Let $\kappa \in \mathbb{N}^{n}, T: \mathbb{C}_{\kappa}\left[z_{1}, \ldots, z_{n}\right] \rightarrow \mathbb{C}\left[z_{1}, \ldots, z_{n}\right]$ be a non-degenerate linear operator and $C_{i}, i \in[n]$, be open circular domains given by $C_{i}=\phi_{i}^{-1}(H)$, $i \in[n]$, where $\phi_{i}, i \in[n]$, are Möbius transformations as in Theorem [6.3. Then $T$ preserves the $\kappa$-Lee-Yang property with respect to $\left\{C_{i}\right\}_{i=1}^{n}$ if and only if either

(a) the polynomial in $2 n$ variables $z_{1}, \ldots, z_{n}, w_{1}, \ldots, w_{n}$ given by

$$
\begin{gathered}
T\left[\prod_{i=1}^{n}\left(\left(a_{i} z_{i}+b_{i}\right)\left(c_{i} w_{i}+d_{i}\right)+\left(a_{i} w_{i}+b_{i}\right)\left(c_{i} z_{i}+d_{i}\right)\right)^{\kappa_{i}}\right] \\
\text { is } C_{1} \times \cdots \times C_{n} \times C_{1} \times \cdots \times C_{n} \text {-stable and } C_{1}^{r} \times \cdots \times C_{n}^{r} \times C_{1}^{r} \times \cdots \times C_{n}^{r} \text {-stable, } \\
\text { or } \begin{array}{r}
\text { (b) the polynomial in } 2 n \text { variables } z_{1}, \ldots, z_{n}, w_{1}, \ldots, w_{n} \text { given by } \\
T\left[\prod_{i=1}^{n}\left(\left(a_{i} z_{i}+b_{i}\right)\left(c_{i} w_{i}+d_{i}\right)-\left(a_{i} w_{i}+b_{i}\right)\left(c_{i} z_{i}+d_{i}\right)\right)^{\kappa_{i}}\right] \\
\text { is } C_{1} \times \cdots \times C_{n} \times C_{1} \times \cdots \times C_{n} \text {-stable and } C_{1}^{r} \times \cdots \times C_{n}^{r} \times C_{1}^{r} \times \cdots \times C_{n}^{r} \text {-stable. }
\end{array}
\end{gathered}
$$

Proof. The proof of the corresponding theorem in the case $n=1$ from [7], namely [7. Theorem 3], extends naturally to the above setting.

\section{Related Results}

A multivariate polynomial is called strictly stable if it is non-vanishing whenever all variables lie in $\bar{H}=\{z \in \mathbb{C}: \operatorname{Im}(z) \geq 0\}$. The arguments in 92 carry over to the closed upper half-plane $\bar{H}$ and yield the following sufficient condition for strict stability preserving.

Theorem 7.1. Let $\kappa \in \mathbb{N}^{n}$ and $T: \mathbb{C}_{\kappa}\left[z_{1}, \ldots, z_{n}\right] \rightarrow \mathbb{C}\left[z_{1}, \ldots, z_{n}\right]$ be a linear operator. If $G_{T}(z, w)$ is a strictly stable polynomial then $T$ preserves strict stability.

One can also check that the sufficiency part of Theorem 6.3 carries over to closed circular domains and thus yields a (sufficient) condition for linear operators $T: \mathbb{C}_{\kappa}\left[z_{1}, \ldots, z_{n}\right] \rightarrow \mathbb{C}\left[z_{1}, \ldots, z_{n}\right]$ to preserve $\bar{C}_{1} \times \cdots \times \bar{C}_{n}$-stability, where $C_{i}$, $i \in[n]$, are arbitrary (open) circular domains. For simplicity we only state here a special case that extends Theorems 7.1 .

Theorem 7.2. Let $\kappa \in \mathbb{N}^{n}, T: \mathbb{C}_{\kappa}\left[z_{1}, \ldots, z_{n}\right] \rightarrow \mathbb{C}\left[z_{1}, \ldots, z_{n}\right]$ a linear operator and $\bar{C}$ a convex closed circular domain given by $\bar{C}=\phi^{-1}(\bar{H})$, where $\phi$ is a Möbius transformation as in 6.1). If

$$
T\left[((a z+b)(c w+d)+(a w+b)(c z+d))^{\kappa}\right]
$$

is $\bar{C}$-stable then $T$ preserves $\bar{C}$-stability. 
As noted in Remark 6.1, for the the closed unit disk $\bar{C}=\overline{\mathbb{D}}$ the symbol used in Theorem 7.2 is just a constant multiple of $T\left[(1+z w)^{\kappa}\right]$ while for a closed half-plane bordering on the origin it is a constant multiple of $G_{T}(z, w)=T\left[(z+w)^{\kappa}\right]$. Note though that the conditions in Theorems 7.1, 7.2 are not necessary. For instance, the identity operator obviously preserves strict stability but its (algebraic) symbol $(z+w)^{\kappa}$ is not strictly stable, cf. (i) in 88 .

\section{Further Directions}

To conclude we mention some of the most appealing cases where Problems 12 remain open:

(i) $\Omega$ is a closed circular domain, to begin with $\Omega=\bar{H}$ (cf. Theorem 7.1);

(ii) $\Omega$ is a strip, e.g., $\Omega=\{z \in \mathbb{C}:|\operatorname{Im}(z)|<a\}, a>0$;

(iii) $\Omega$ is a sector, e.g., $\Omega=\left\{z \in \mathbb{C}: \frac{\pi}{2}-\alpha<\arg (z)<\frac{\pi}{2}+\alpha\right\}, \alpha \in\left(0, \frac{\pi}{2}\right)$;

(iv) $\Omega$ is a closed or open strip or (double) sector.

Finally, from a complex analytic perspective and its many potential applications [10] it would be quite useful to establish analogs of these results for transcendental entire functions in one or several variables.

\section{ACKNOWLEDGMENTS}

We wish to thank the Isaac Newton Institute for Mathematical Sciences, University of Cambridge, for generous support during the programme "Combinatorics and Statistical Mechanics" (January-June 2008), where this work, 6] and [11] were presented in a series of lectures. Special thanks are due to the organizers and the participants in this programme for numerous stimulating discussions. We would also like to thank Alex Scott, Béla Bollobás and Richard Kenyon for the opportunities to announce these results in June-July 2008 through talks given at the Mathematical Institute, University of Oxford, the Centre for Mathematical Sciences, University of Cambridge, and the programme "Recent Progress in Two-Dimensional Statistical Mechanics", Banff International Research Station, respectively.

\section{REFERENCES}

[1] T. Asano, Theorems on the partition functions of the Heisenberg ferromagnets, J. Phys. Soc. Japan 29 (1970), 350-359.

[2] M. F. Atiyah, R. Bott, L. Gårding, Lacunas for hyperbolic differential operators with constant coefficients I, Acta Math. 124 (1970), 109-189.

[3] B. Beauzamy, On complex Lee and Yang polynomials, Comm. Math. Phys. 182 (1996), 177184.

[4] M. Biskup, C. Borgs, J. T. Chayes, R. Kotecky, Partition function zeros at first-order phase transitions: Pirogov-Sinai theory, J. Statist. Phys. 116 (2004), 97-155.

[5] M. Biskup, C. Borgs, J. T. Chayes, L. J. Kleinwaks, R. Kotecky, Partition function zeros at first-order phase transitions: A general analysis, Comm. Math. Phys. 251 (2004), 79-131.

[6] J. Borcea, P. Brändén, The Lee-Yang and Pólya-Schur programs. II. Theory of stable polynomials and applications, arXiv:0809.3087.

[7] J. Borcea, P. Brändén, Pólya-Schur master theorems for circular domains and their boundaries, Ann. of Math., to appear; arXiv:math/0607416

[8] J. Borcea, P. Brändén, Applications of stable polynomials to mixed determinants: Johnson's conjectures, unimodality, and symmetrized Fischer products, Duke Math. J. 143 (2008), 205-223.

[9] J. Borcea, P. Brändén, Multivariate Pólya-Schur classification problems in the Weyl algebra, arXiv:math/0606360. 
[10] J. Borcea, P. Brändén, G. Csordas, V. Vinnikov, Pólya-Schur-Lax problems: hyperbolicity and stability preservers, Workshop Report, American Institute of Mathematics, Palo Alto, CA, May-June 2007; http://www.aimath.org/pastworkshops/polyaschurlax.html

[11] J. Borcea, P. Brändén, T. M. Liggett, Negative dependence and the geometry of polynomials, J. Amer. Math. Soc. 22 (2009), 521-567; arXiv:0707.2340

[12] P. Brändén, Polynomials with the half-plane property and matroid theory, Adv. Math. 216 (2007), 302-320.

[13] Y. Choe, J. Oxley, A. D. Sokal, D. G. Wagner, Homogeneous multivariate polynomials with the half-plane property, Adv. Appl. Math. 32 (2004), 88-187.

[14] T. Craven, G. Csordas, Composition theorems, multiplier sequences and complex zero decreasing sequences, in "Value Distribution Theory and Its Related Topics", G. Barsegian, I. Laine, C. C. Yang (Eds.), pp. 131-166, Kluver Press, 2004.

[15] T. Craven, G. Csordas, W. Smith, The zeros of derivatives of entire functions and the PólyaWiman conjecture, Ann. of Math. (2) 125 (1987), 405-431.

[16] T. Craven, G. Csordas, Multiplier sequences for fields, Illinois J. Math. 21 (1977), 801-817.

[17] G. Csordas, Linear operators and the distribution of zeros of entire functions, Complex Var. Elliptic Equ. 51 (2006), 625-632.

[18] A. Edrei, Power series having partial sums with zeros in a half-plane, Proc. Amer. Math. Soc. 9 (1958), 320-324.

[19] S. Fisk, Polynomials, roots, and interlacing. Versions 1-2, http://www.bowdoin.edu/fisk/, $\mathrm{xx}+700 \mathrm{pp}$

[20] J. H. Grace, The zeros of a polynomial, Proc. Cambridge Philos. Soc. 11 (1902), 352-357.

[21] L. Gårding, An inequality for hyperbolic polynomials, J. Math. Mech. 8 (1959), 957-965.

[22] O. J. Heilmann, E. H. Lieb, Theory of monomer-dimer systems, Comm. Math. Phys. 25 (1972), 190-232.

[23] A. Hinkkanen, Schur products of certain polynomials, in "Lipa's Legacy: Proceedings of the Bers Colloquium", J. Dodziuk, L. Keenin (Eds.), Contemp. Math. 211, Amer. Math. Soc., Providence, RI, 1997, pp. 285-295.

[24] L. Hörmander, On a theorem of Grace, Math. Scand. 2 (1954), 55-64.

[25] L. Hörmander, Notions of Convexity. Progr. Math. 127. Birkhäuser, Boston, MA, 1994.

[26] A. Iserles, S. P. Nørsett, E. B. Saff, On transformations and zeros of polynomials, Rocky Mountain J. Math. 21 (1991), 331-357.

[27] R. Kenyon, A. Okounkov, S. Sheffield, Dimers and amoebae, Ann. of Math. (2) 163 (2006), 1019-1056.

[28] E. Laguerre, Fonctions du genre zéro et du genre un, C. R. Acad. Sci. Paris 95 (1882), 828-831.

[29] P. D. Lax, Differential equations, difference equations and matrix theory, Comm. Pure Appl. Math. 6 (1958), 175-194.

[30] G. F. Lawler, O. Schramm, W. Werner, Values of Brownian intersection exponents. I. Halfplane exponents, Acta Math. 187 (2001), 237-273; II. Plane exponents, ibid., 275-308.

[31] T. D. Lee, C. N. Yang, Statistical theory of equations of state and phase transitions. II. Lattice gas and Ising model, Phys. Rev. 87 (1952), 410-419.

[32] B. Ja. Levin, Distribution of zeros of entire functions. Transl. Math. Monogr. Vol. 5, Amer. Math. Soc., Providence, R.I., 1980.

[33] E. H. Lieb, A. D. Sokal, A General Lee-Yang Theorem for One-Component and Multicomponent Ferromagnets, Comm. Math. Phys. 80 (1981), 153-179.

[34] T. M. Liggett, Distributional Limits for the Symmetric Exclusion Process, to appear in Stoch. Proc. Appl., arXiv:0710.3606

[35] M. Marden, The Geometry of the Zeros of a Polynomial in a Complex Variable. Math. Surveys Vol. 3, Amer. Math. Soc., New York, NY, 1949.

[36] C. M. Newman, Gaussian correlation inequalities for ferromagnets, Z. Wahrscheinlichkeitstheorie und Verw. Gebiete 33 (1975), 75-93.

[37] C. M. Newman, Inequalities for Ising models and field theories which obey the Lee-Yang theorem, Comm. Math. Phys. 41 (1975), 1-9.

[38] C. M. Newman, Zeros of the partition function for generalized Ising systems, Comm. Pure Appl. Math. 27 (1974), 143-159.

[39] G. Pólya, Bemerkung über die Integraldarstellung der Riemannsche $\xi$-Funktion, Acta Math. 48 (1926), 305-317. 
[40] G. Pólya, Collected Papers, Vol. II: Location of Zeros. Mathematicians of our Time. Vol. 8 (R. P. Boas, ed.), The MIT Press, Cambridge, MA, 1974.

[41] G. Pólya, I. Schur, Über zwei Arten von Faktorenfolgen in der Theorie der algebraischen Gleichungen, J. Reine Angew. Math. 144 (1914), 89-113.

[42] G. Pólya, G. Szegö, Problems and Theorems in Analysis. Vol. II, Springer, Berlin, 1976.

[43] Q. I. Rahman, G. Schmeisser, Analytic Theory of Polynomials. London Math. Soc. Monogr. (N. S.) Vol. 26, Oxford Univ. Press, New York, NY, 2002.

[44] D. Ruelle, Zeros of graph-counting polynomials, Comm. Math. Phys. 200 (1999), 43-56.

[45] D. Ruelle, Grace-like polynomials, Foundations of computational mathematics (Hong Kong, 2000), pp. 405-421, World Sci. Publ. Co. Inc., River Edge, NJ, 2002.

[46] D. Ruelle, Is our mathematics natural? The case of equilibrium statistical mechanics, Bull. Amer. Math. Soc. (N.S.) 19 (1988), 259-268.

[47] D. Ruelle, Some remarks on the location of zeroes of the partition function for lattice systems, Comm. Math. Phys. 31 (1973), 265-277.

[48] D. Ruelle, Extension of the Lee-Yang circle theorem, Phys. Rev. Lett. 26 (1971), 303-304.

[49] D. Ruelle, Statistical mechanics: Rigorous results. Reprint of the 1989 edition, World Sci. Publ. Co. Inc., River Edge, NJ, 1999.

[50] I. Schur, Zwei Sätze über algebraische Gleichungen mit lauter reellen Wurzeln, J. Reine Angew. Math. 144 (1923), 75-88.

[51] A. D. Scott, A. D. Sokal, The repulsive lattice gas, the independent-set polynomial, and the Lovász local lemma, J. Stat. Phys. 118 (2005), 1151-1261; arXiv:cond-mat/0309352

[52] S. Smirnov, W. Werner, Critical exponents for two-dimensional percolation, Math. Res. Lett. 8 (2001), 729-744.

[53] A. D. Sokal, The multivariate Tutte polynomial (alias Potts model) for graphs and matroids, in "Surveys in Combinatorics, 2005" (B. S. Webb, ed.), Cambridge Univ. Press, Cambridge, 2005 ; arXiv:math.CO/0503607.

[54] A. D. Sokal, Chromatic roots are dense in the whole complex plane, Combin. Probab. Comput. 13 (2004), 221-261.

[55] O. Szász, On sequences of polynomials and the distribution of their zeros, Bull. Amer. Math. Soc. 49 (1943), 377-383.

[56] G. Szegö, Bemerkungen zu einem Satz von J. H. Grace über die Wurzeln algebraischer Gleichungen, Math. Z. 13 (1922), 28-55.

[57] D. G. Wagner, Weighted enumeration of spanning subgraphs with degree constraints, preprint arXiv:0803.1659.

[58] J. L. Walsh, On the location of the roots of certain types of polynomials, Trans. Amer. Math. Soc. 24 (1922), 163-180.

[59] C. N. Yang, T. D. Lee, Statistical theory of equations of state and phase transitions. I. Theory of Condensation, Phys. Rev. 87 (1952), 404-409.

Department of Mathematics, Stockholm University, SE-106 91 Stockholm, Sweden

E-mail address: julius@math.su.se

Department of Mathematics, Royal Institute of Technology, SE-100 44 Stockholm, SwEDEN

E-mail address: pbranden@math.kth.se 